\title{
Boundary value problems for periodic analytic functions
}

Pei Dang ${ }^{1}$, Jinyuan Du ${ }^{2,3 *}$ and Tao Qian ${ }^{4}$

\section{"Correspondence:}

jydu@whu.edu.cn

${ }^{2}$ School of Science, Linyi University, Linyi, Shandong 276000, P.R. China

${ }^{3}$ Department of Mathematics,

Wuhan University, Wuhan, 430072,

P.R. China

Full list of author information is

available at the end of the article

\begin{abstract}
In this paper boundary value problems for periodic analytic functions are discussed. We first introduce definitions of principal part and order at $\pm \infty i$ for periodic analytic functions through detailed analysis. Then Riemann boundary value problems for periodic analytic functions with finite order at $\pm \infty i$ are formulated. Based on those, by using the exponential conformal mapping, Riemann boundary value problems for periodic sectionally holomorphic functions with periodic closed and periodic quasi-closed contours as their jump curves are solved. The method that we use here has computational advantages compared with the tangent mapping one used in solving the classical problems. Several types of Hilbert boundary value problems on the real axis and the circumferences for periodic analytic functions are also solved.
\end{abstract}

MSC: 30E25; 45E05

Keywords: principal part; order at $\pm \infty$ i; periodic Riemann boundary value problem; periodic Hilbert boundary value problem

\section{Introduction}

The theory of boundary value problems for analytic functions is an important branch of complex analysis. It has ample applications due to the fact that many practical problems in mechanics, physics, and engineering may be converted to boundary value problems or singular integral equations [1-6]. Boundary value problems for analytic functions have been systematically investigated in the related literature (see, for instance, [7-9]). In the monograph [7] Lu first introduced the so-called periodic Riemann boundary value problems (PR problems) and periodic Hilbert boundary value problems ( $\mathrm{PH}$ problems) motivated from the periodic problems in plane elasticity. More general formulations of such problems are for automorphic functions which were first studied by Gakhov and Chibrikova $[9,10]$. From the practical point of view, the periodic boundary value problems are considerably important. In particular, by using the solutions and methods for PR problems and $\mathrm{PH}$ problems one can effectively solve a number of periodic problems in plane elasticity [11-13].

In [7], Lu mainly discussed PR problems with periodic closed contour as the jump curve and $\mathrm{PH}$ problems on the real axis for bounded solutions. He transferred periodic boundary value problems to standard Riemann boundary problems and Hilbert boundary problems by the tangent conformal mapping. The theme of unbounded solutions at the infinity has not been well addressed, although some results were predicted [7]. The predicted results,

( 2015 Dang et al. This article is distributed under the terms of the Creative Commons Attribution 4.0 International License (http://creativecommons.org/licenses/by/4.0/), which permits unrestricted use, distribution, and reproduction in any medium, provided you give appropriate credit to the original author(s) and the source, provide a link to the Creative Commons license, and indicate if changes were made. 
however, are not accurate, for there is no detailed and rigorous analysis for the behavior of the solutions at the infinity.

In the present paper, we will discuss in depth three different types of problems: (i) the Riemann boundary value problem for periodic sectionally holomorphic functions with periodic closed contour or quasi-closed contour as jump curves; (ii) the Hilbert boundary value problem on the real axis for the periodic holomorphic function in the upper half-plane; and (iii) the Hilbert boundary value problem on periodic circumferences for periodic holomorphic functions in the disks, being the interior regions of a set of periodic circumferences. In the next section, we shall introduce definitions of the principal part and order at the infinity for periodic holomorphic functions by using the exponential conformal mapping. Then Riemann boundary value problems for periodic holomorphic functions with finite orders at $\pm \infty i$ are presented in Section 3. We transfer the PR problem to a standard Riemann boundary value problem by the exponential conformal mapping, and the solution formula and a set of conditions for the solvability are obtained. In Section 4, the periodic Hilbert boundary value problem on the real axis and on periodic circumferences are discussed, respectively, in two ways. The first is the so-called reflex extension and the second is the so-called regular factor method.

Periodic Riemann boundary value problems and periodic Hilbert boundary value problems have applications to global asymptotic analysis for orthogonal polynomials on the real axis. To keep the present paper within a reasonable bound the application aspect will be postponed to a forthcoming paper.

\section{Order of periodic analytic functions at the infinity}

Assume

$$
L=\sum_{k=-\infty}^{+\infty} L_{k}
$$

is a set of smooth closed contours, non-intersecting to each other, with the same shape and size, arranged horizontally with period $a \pi(a>0)$ and oriented counter-clockwise (see Figure 1), which is called a periodic closed contour. The interior region of $L_{k}$ is denoted by $S_{k}^{+}$and the exterior of $L$ by $S^{-}$. We may assume $O \in S_{0}^{+}$and $\pm a \pi / 2 \in S^{-}$, which is always possible through a translation of the axes if necessary.

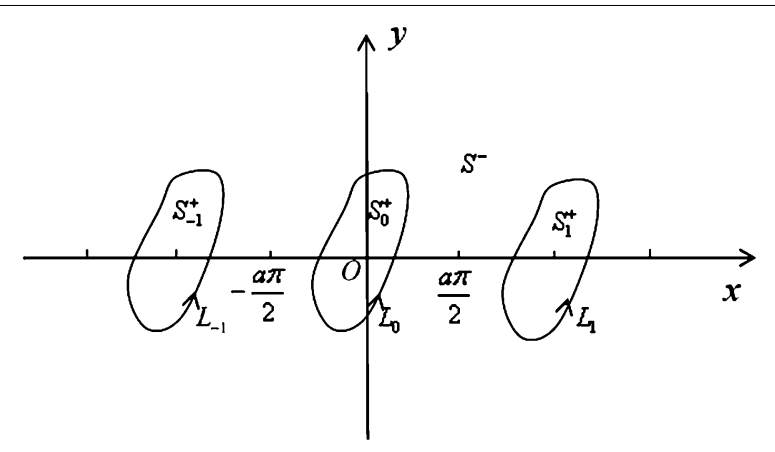

Figure 1 Periodic closed contour, its interior and exterior regions. 
A set $\mathscr{S}$ is called the periodic set with the period $a \pi(a>0)$, if $z \pm a \pi \in \mathscr{S}$ for any $z \in \mathscr{S}$. So, $S^{-}, S^{+}$, and $L$ are all periodic sets with the period $a \pi$, where

$$
S^{+}=\bigcup_{k=-\infty}^{+\infty} S_{k}^{+} .
$$

The PR problem is formulated as follows (see [7]): Find a periodic sectionally holomorphic function $\Phi(z)$ with $L$ as the jump curve such that

$$
\Phi^{+}(t)=G(t) \Phi^{-}(t)+g(t), \quad t \in L,
$$

where $G$ and $g$ are given on $L$ with the period $a \pi$, that is,

$$
G(t+a \pi)=G(t), \quad g(t+a \pi)=g(t)
$$

satisfying the normal type condition

$$
G(t) \neq 0, \quad t \in L,
$$

and the Hölder conditions

$$
G \in H(L), \quad g \in H(L) .
$$

In general, infinity $\infty$ should not be an isolated singular point of the solution of the PR problem (if any). In relation to this, its principal part and order at infinity $\infty$ are not well defined in the classical sense. Let $\mathcal{C}$ be the complex plane, and, for $z \in \mathcal{C}, x=\operatorname{Re} z$, and $y=\operatorname{Im} z$. For any $h \in \mathcal{R}$, we call the sets

$$
N(+\infty i, h)=\{z: y=\operatorname{Im} z>h\}, \quad N(-\infty i, h)=\{z: y=\operatorname{Im} z<h\}
$$

neighborhoods of $+\infty i$ and $-\infty i$, respectively. The exponential conformal mapping

$$
w=e^{\frac{2 i z}{a}}, \quad z \in \mathcal{C},
$$

maps, respectively, the upper strip region and the lower strip region

$$
\begin{aligned}
& N_{h}^{+}=\left\{z: \operatorname{Im} z>h,-\frac{1}{2} a \pi \leq \operatorname{Re} z \leq \frac{1}{2} a \pi\right\}, \\
& N_{h}^{-}=\left\{z: \operatorname{Im} z<h,-\frac{1}{2} a \pi \leq \operatorname{Re} z \leq \frac{1}{2} a \pi\right\}
\end{aligned}
$$

into the interior and exterior of the circle $\left\{w:|w|=e^{-2 h / a}\right\}$, maps the infinity accumulation point $z=+\infty i$ of $N_{h}^{+}$and the infinity accumulation point $z=-\infty i$ of $N_{h}^{-}$to $w=0$ and $w=\infty$, and maps the straight lines $\operatorname{Re} z=-a \pi / 2$ and $\operatorname{Re} z=a \pi / 2$ into the upper and lower bank of the cut $(-\infty, 0)$. 
Let $\Phi^{+}(z)$ be a holomorphic function with the period $a \pi$ on the neighborhood of $+\infty i$, denoted by $\Phi^{+} \in \mathcal{A}_{a \pi}^{+}$. Then

$$
\Phi_{*}^{+}(w)=\Phi^{+}(z)=\Phi^{+}\left(-\frac{a i}{2} \ln w\right), \quad 0<|w|<e^{-\frac{2 h}{a}},
$$

is well defined and analytic by the Painlevé theorem, where the logarithm function $\ln w$ is the principal branch in the complex plane cutting along $(-\infty, 0]$, i.e.,

$$
\ln w=\ln |w|+i \arg (w) \quad(-\pi \leq \arg (w)<\pi), w \neq 0,
$$

which is the inverse mapping of the restriction of the exponential mapping (2.8) on the strip region $S_{0}=\{z: \operatorname{Im} z>0,-\pi \leq \operatorname{Re} z<\pi\}$. Thus, $\Phi_{*}^{+}$has a Laurent expansion, then we know easily that there is also the unique expansion in the series form for $\Phi^{+}[14,15]$, that is,

$$
\Phi^{+}(z)=\sum_{-\infty}^{+\infty} a_{j} e^{i j z} \quad(\operatorname{Im} z>h)
$$

where

$$
\begin{aligned}
& a_{j}=\frac{1}{2 \pi} \int_{\Upsilon} \Phi^{+}(z) e^{-\frac{i j z}{a}} \mathrm{~d} z, \\
& \Upsilon:=\left\{z: z=x+i r,-\frac{a \pi}{2} \leq x \leq \frac{a \pi}{2}, r \text { is an arbitrary constant greater than } h\right\} \\
& \text { or } \Upsilon:=\left\{z: z=x+i h,-\frac{a \pi}{2} \leq x \leq \frac{a \pi}{2}\right\}, \quad \text { while } \Phi^{+} \text {is continuous to it. }
\end{aligned}
$$

If the expansion in (2.12) is

$$
\Phi^{+}(z)=\sum_{j=m}^{+\infty} a_{-j} e^{-\frac{i j z}{a}} \quad \text { with } a_{-m} \neq 0, z \in N_{h}^{+}
$$

then we say that $\Phi^{+}$has the pole of order $m$ at $z=+\infty i$, or simply, order $m$, denoted as $\operatorname{eOrd}\left[\Phi^{+}\right](+\infty i)=m$, which is just equivalent to that its associated function $\Phi_{*}^{+}$has the pole of order $m$ at $w=0$ denoted as $\operatorname{Ord}\left[\Phi_{*}^{+}\right](0)=m$. We agree that the pole of order $m$ is just the zero point of order $-m$. Thus, we has

$$
\operatorname{eOrd}\left[\Phi^{+}\right](+\infty i)=m \quad \Longleftrightarrow \quad \operatorname{Ord}\left[\Phi_{*}^{+}\right](0)=m
$$

If $\Phi^{-}(z)$ is a holomorphic function with the period $a \pi$ on the neighborhood of $-\infty i$, denoted by $\Phi^{-} \in \mathcal{A}_{a \pi}^{-}$, similarly, it also has the unique expansion in the series form

$$
\Phi^{-}(z)=\sum_{-\infty}^{+\infty} b_{j} e^{\frac{i j z}{a}} \quad(\operatorname{Im} z<h)
$$


where

$$
\begin{aligned}
& b_{j}=\frac{1}{2 \pi} \int_{\Upsilon} \Phi^{-}(z) e^{-\frac{i j z}{a}} \mathrm{~d} z, \\
& \Upsilon:=\left\{z: z=x+i r,-\frac{a \pi}{2} \leq x \leq \frac{a \pi}{2}, r \text { is an arbitrary constant smaller than } h\right\} \\
& \text { or } \Upsilon:=\left\{z: z=x+i h,-\frac{a \pi}{2} \leq x \leq \frac{a \pi}{2}\right\}, \quad \text { while } \Phi^{-} \text {is continuous to it. }
\end{aligned}
$$

In particular, if

$$
\Phi^{-}(z)=\sum_{j=m}^{+\infty} b_{j} e^{\frac{i j z}{a}} \quad \text { with } b_{m} \neq 0, z \in N_{h}^{-},
$$

we say that $\Phi^{-}$has the pole of order $m$ at $z=-\infty i$, denoted as $\operatorname{eOrd}\left[\Phi^{-}\right](-\infty i)=m$, which is just equivalent to that its associated function

$$
\left[\Phi_{*}^{-}\right](w)=\Phi^{-}(z)=\Phi^{-}\left(-\frac{a i}{2} \ln w\right), \quad|w|>e^{-\frac{2 h}{a}},
$$

where the mapping (2.11) has the pole of order $m$ at $w=\infty$, denoted as $\operatorname{Ord}\left[\Phi_{*}^{-}\right](\infty)=m$, i.e.,

$$
\operatorname{eOrd}\left[\Phi^{-}\right](-\infty i)=m \quad \Longleftrightarrow \quad \operatorname{Ord}\left[\Phi_{*}^{-}\right](\infty)=m
$$

\section{Periodic Riemann boundary value problems}

In the section, we give the formulation and transformation of periodic Riemann boundary value problems. Then both the expression of the solution and the condition of solvability are obtained in closed form.

\subsection{Formulation of the problem}

In PR problem (2.3), if $\Phi$ is required to have at most order $\mathrm{n}$ at $+\infty i$ and order $m$ at most at $-\infty i$, then the problem is denoted by $P R_{m, n}$.

$P R_{m, n}$ problem: Find a periodic sectionally holomorphic function $\Phi(z)$ with $L$ as the jump curve such that

$$
\left\{\begin{array}{l}
\Phi^{+}(t)=G(t) \Phi^{-}(t)+g(t), \quad t \in L \\
\mathrm{eOrd}[\Phi](+\infty i) \leq n \\
\mathrm{eOrd}[\Phi](-\infty i) \leq m
\end{array}\right.
$$

where $G$ and $g$ are defined on $L$ satisfying (2.4), (2.5), and (2.6). $L$ is the periodic closed contour given in the last section. In addition, $L$ can also be the periodic quasi-closed contour given below.

A set of smooth open $\operatorname{arcs} L_{j}=\widehat{a_{j} a_{j+1}}(j=0, \pm 1, \pm 2, \ldots)$ is called a periodic quasi-closed contours, if the $L_{j}$ have the same shape and size, arranged horizontally with period $a \pi$ $(a>0), a_{j}=\left(j-\frac{1}{2}\right) a \pi$ and the tangents of $L_{j}$ at their ends are parallel. For concreteness, we assume that the position of the origin $O$ cannot be below the curve $L$. Such $L$ divides the complex plane $\mathcal{C}$ into two parts, the upper one denoted by $S^{+}$and the lower one by $S^{-}$ 


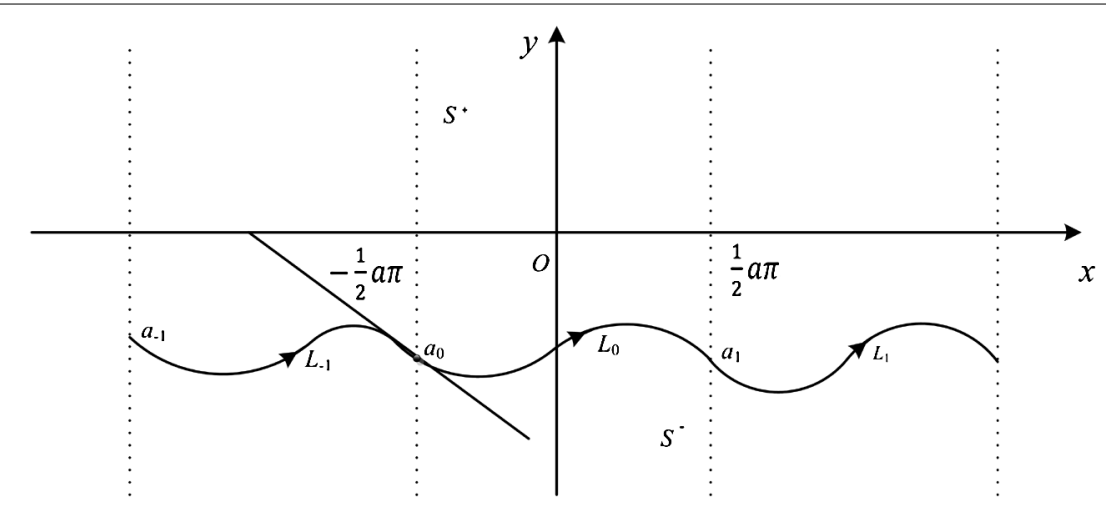

Figure 2 A periodic quasi-closed contour.

(see Figure 2). The particular case $L_{j}=\left[\left(j-\frac{1}{2}\right) a \pi,\left(j+\frac{1}{2}\right) a \pi\right]$ is worth paying attention to, for in that case $L$ reduces to the real axis.

\subsection{Transformation of the problem}

Let $L$ be a periodic closed contour or a periodic quasi-closed contour. The following discussions and even all the results for the two cases are essentially the same. For example, under the mapping (2.8), the image of $L_{0}$ on the $w$-plane, denoted by $\Gamma$, is a smooth closed contour surrounding $w_{0} . \Gamma$ divides the $w$-plane into the interior region $\Sigma^{+}$and the exterior region $\Sigma^{-}$(see Figure 3 and Figure 4), which are, respectively, the images of the strip regions

$$
\begin{aligned}
& S_{0}^{+}=\left\{z: z \in S^{+},-\frac{1}{2} a \pi \leq \operatorname{Re}(z) \leq \frac{1}{2} a \pi\right\} \\
& S_{0}^{-}=\left\{z: z \in S^{-},-\frac{1}{2} a \pi \leq \operatorname{Re}(z) \leq \frac{1}{2} a \pi\right\} .
\end{aligned}
$$

In the sequel, for simplicity, we will take

$$
w_{0}= \begin{cases}1, & \text { when } 1 \in \Sigma^{+}(\text {Figure } 3), \\ 0, & \text { when } 1 \notin \Sigma^{+}(\text {Figure } 4) .\end{cases}
$$

Remark 3.1 When $L$ is a periodic quasi-closed contour, it is possible that $1 \notin \Sigma^{+}$. For example, $L$ can be the real axis. Of course, we may always take $1 \in \Sigma^{+}$by a translation transform.

As before, we easily see that, if $\Phi(z)$ is a periodic sectionally holomorphic function with $L$ being the jump curve, then, under the mapping (2.8) or (2.11), its associated function,

$$
\Phi_{*}(w)=\Phi\left(-\frac{a i}{2} \ln w\right), \quad w \in \mathcal{C} \backslash \Gamma
$$

is a sectionally holomorphic function, where $\Gamma$ is the jump curve and

$$
\Phi_{*}^{ \pm}(\tau)=\Phi^{ \pm}(t) \quad \text { with } \tau=e^{\frac{2 i t}{a}} \in \Gamma(t \in L) .
$$

Therefore, $\Phi_{*}$ is the solution of the following boundary value problem. 

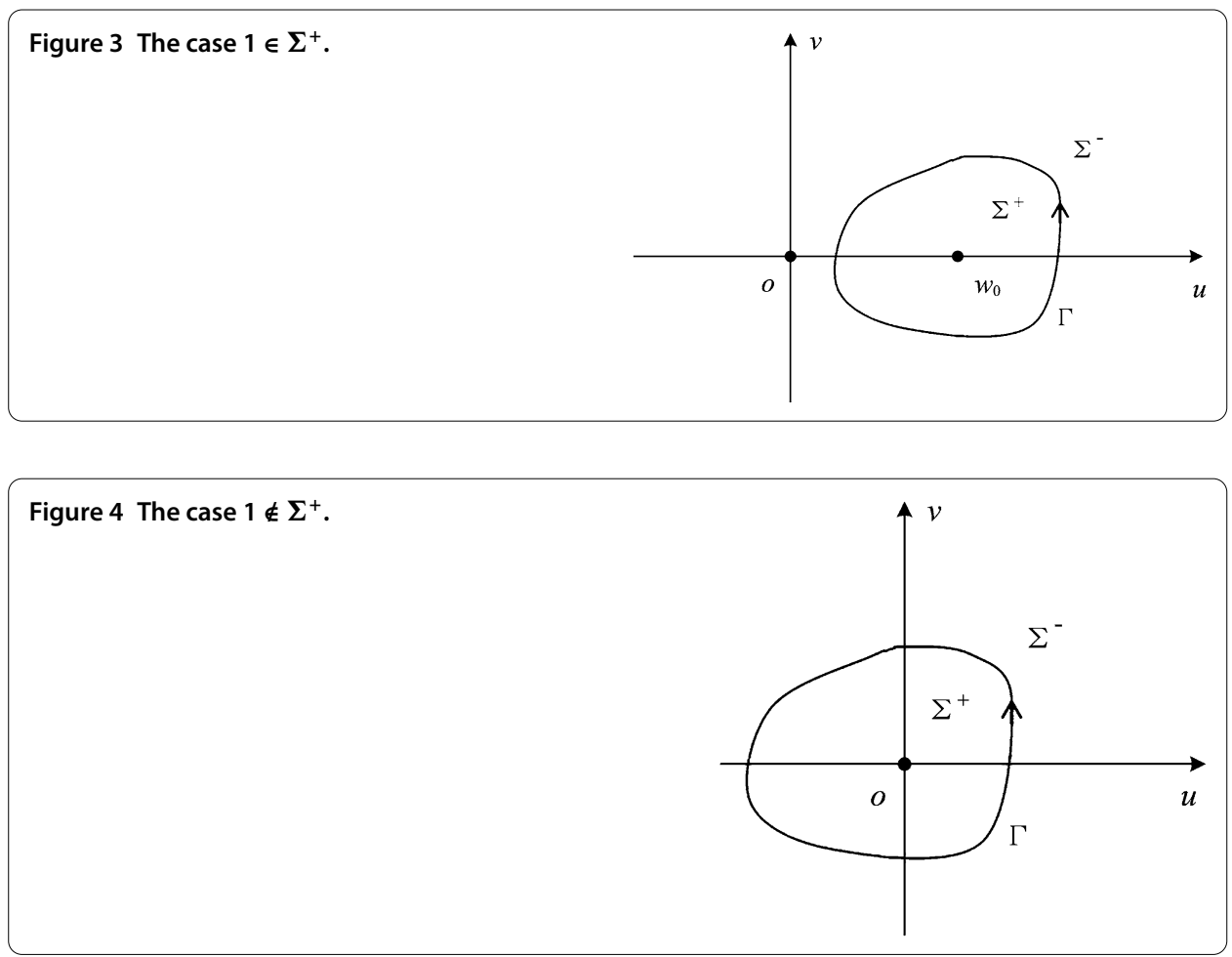

$R_{m, n}^{*}$ problem: Find a sectionally meromorphic function $\Phi_{*}(w)$ having possible pole point at $w=0$, with $\Gamma$ as the jump curve, such that

$$
\left\{\begin{array}{l}
\Phi_{*}^{+}(\tau)=G_{*}(\tau) \Phi_{*}^{-}(\tau)+g_{*}(\tau), \quad \tau \in \Gamma \\
\operatorname{Ord}\left[\Phi_{*}\right](0) \leq n \\
\operatorname{Ord}\left[\Phi_{*}\right](\infty) \leq m
\end{array}\right.
$$

where

$$
G_{*}(\tau)=G\left(-\frac{a i}{2} \ln \tau\right), \quad g_{*}(\tau)=g\left(-\frac{a i}{2} \ln \tau\right), \quad \tau \in \Gamma,
$$

with the mapping (2.11). Obviously,

$$
G_{*}(\tau) \neq 0, \quad \tau \in \Gamma .
$$

It is easy to prove that

$$
G_{*} \in H(\Gamma), \quad g_{*} \in H(\Gamma) .
$$

Conversely, if $\Phi_{*}$ is the solution of $R_{m, n}^{*}$ problem (3.6), then

$$
\Phi(z)=\Phi_{*}\left(e^{\frac{2 i z}{a}}\right), \quad z \in \mathcal{C} \backslash L,
$$

is the solution of $P R_{m, n}$ problem (3.1). In fact, it is obvious that the above $\Phi$ is a periodic sectionally holomorphic function whose jump curve is $L$ such that (3.5), (2.15), and (2.20) hold. It is easy to verify that $\Phi$ given by (3.10) is the solution of $P R_{m, n}$ problem (3.1). 
We summarize the above discussion as follows.

Lemma 3.1 Under the relationship (3.4) or (3.10), $P R_{m, n}$ problem (3.1) is equivalent to $R_{m, n}^{*}$ problem (3.6).

Let

$$
\begin{aligned}
& \Phi_{\sharp}(w)=w^{n} \Phi_{*}(w), \quad w \in \mathcal{C} \backslash \Gamma, \\
& G_{\sharp}(\tau)=G_{*}(\tau) \quad \text { and } \quad g_{\sharp}(\tau)=\tau^{n} g_{*}(\tau), \quad \tau \in \Gamma .
\end{aligned}
$$

Then we transfer the $R_{m, n}^{*}$ problem to the following Riemann boundary value problem.

$R_{m+n}$ problem: Find a sectionally holomorphic function $\Phi_{\sharp}(w)$, with $\Gamma$ as the jump curve, such that

$$
\left\{\begin{array}{l}
\Phi_{\sharp}^{+}(\tau)=G_{\sharp}(\tau) \Phi_{\sharp}^{-}(\tau)+g_{\sharp}(\tau), \quad \tau \in \Gamma, \\
\operatorname{Ord}\left[\Phi_{\sharp}\right](\infty) \leq m+n .
\end{array}\right.
$$

This is a classical Riemann boundary value problem $[7,8]$.

Lemma 3.2 Under the relationships (3.11) and (3.12), $R_{m, n}^{*}$ problem (3.6) is equivalent to $R_{m+n}$ problem (3.13). More specifically, if $\Phi_{*}$ is the solution of $R_{m, n}^{*}$ problem (3.6), then $\Phi_{\sharp}$ is the solution of $R_{m+n}$ problem (3.13). Conversely, if $\Phi_{\sharp}$ is the solution of $R_{m+n}$ problem (3.13), then $\Phi_{*}$ is the solution of $R_{m, n}^{*}$ problem (3.6).

\subsection{Solution of problem}

We call

$$
\kappa=\operatorname{Ind}_{L_{0}} G(t)=\frac{1}{2 \pi}[\arg G(t)]_{L_{0}}
$$

the index of $G$ or the index of $P R_{m, n}$ problem (3.1). Obviously, it is just the index of $G_{*}=G_{\sharp}$ or $R_{m, n}^{*}$ problem (3.6) and $R_{m+n}$ problem (3.13), i.e.,

$$
\kappa=\operatorname{Ind}_{\Gamma} G_{*}(\tau)=\frac{1}{2 \pi}\left[\arg G_{*}(\tau)\right]_{\Gamma} \equiv \frac{1}{2 \pi}\left[\arg G_{\sharp}(\tau)\right]_{\Gamma}=\operatorname{Ind}_{\Gamma} G_{\sharp}(\tau) .
$$

$R_{m+n}$ problem (3.13) is an ordinary Riemann boundary value problem. From [7, 8], we know that, when $m+n+\kappa \geq-1$, the general solution of $R_{m+n}$ problem (3.13) is

$$
\Phi_{\sharp}(w)=X_{\sharp}(w)\left[\Psi_{\sharp}(w)+P_{m+n+\kappa}(w)\right], \quad w \in \mathcal{C} \backslash \Gamma,
$$

where $P_{r}$ is an arbitrary polynomial of degree not greater than $r\left(P_{r} \equiv 0\right.$ if $\left.r<0\right)$, denoted as $P_{r} \in \Pi_{r}$. The canonical function

$$
X_{\sharp}(w)= \begin{cases}e^{\Gamma_{\sharp}(w)}, & w \in \Sigma^{+}, \\ \left(w-w_{0}\right)^{-\kappa} e^{\Gamma_{\sharp}(w)}, & w \in \Sigma^{-},\end{cases}
$$


where $w_{0}$ is given by (3.3),

$$
\begin{aligned}
\Gamma_{\sharp}(w) & =\frac{1}{2 \pi i} \int_{\Gamma} \frac{\log \left[\left(\tau-w_{0}\right)^{-\kappa} G_{\sharp}(\tau)\right]}{\tau-w} \mathrm{~d} \tau-C \\
& =\frac{1}{2 \pi i} \int_{\Gamma} \frac{\log \left[\left(\tau-w_{0}\right)^{-\kappa} G_{*}(\tau)\right]}{\tau-w} \mathrm{~d} \tau-C,
\end{aligned}
$$

with an arbitrary complex constant $C$ and an arbitrary branch of the logarithm, and

$$
\Psi_{\sharp}(w)=\frac{1}{2 \pi i} \int_{\Gamma} \frac{g_{\sharp}(\tau)}{X_{\sharp}^{+}(\tau)(\tau-w)} \mathrm{d} \tau=\frac{1}{2 \pi i} \int_{\Gamma} \frac{\tau^{n} g_{*}(\tau)}{X_{\sharp}^{+}(\tau)(\tau-w)} \mathrm{d} \tau, \quad w \in \mathcal{C} \backslash \Gamma .
$$

When $m+n+\kappa<-1, R_{m+n}$ problem (3.13) has the unique solution

$$
\Phi_{\sharp}(w)=X_{\sharp}(w) \Psi_{\sharp}(w), \quad w \in \mathcal{C} \backslash \Gamma,
$$

if and only if the following $-(m+n+\kappa+1)$ conditions hold:

$$
\int_{\Gamma} \frac{g_{\sharp}(\tau)}{X_{\sharp}^{+}(\tau)} \tau^{j} \mathrm{~d} \tau=0, \quad j=0,1, \ldots,-(m+n+\kappa)-2,
$$

i.e.,

$$
\int_{\Gamma} \frac{g_{*}(\tau)}{X_{\sharp}^{+}(\tau)} \tau^{j} \mathrm{~d} \tau=0, \quad j=n, n+1, \ldots,-m-\kappa-2 .
$$

Remark 3.2 $\Psi_{\sharp}$ in (3.19) may be rewritten as

$$
\begin{aligned}
\Psi_{\sharp}(w) & =\frac{w^{n}}{2 \pi i} \int_{\Gamma} \frac{g_{*}(\tau)}{X_{\sharp}^{+}(\tau)(\tau-w)} \mathrm{d} \tau+\frac{1}{2 \pi i} \int_{\Gamma} \frac{g_{*}(\tau)}{X_{\sharp}^{+}(\tau)} \frac{\tau^{n}-w^{n}}{\tau-w} \mathrm{~d} \tau \\
& = \begin{cases}w^{n} \Omega_{\sharp}(w)+\triangleright_{n-1}^{\sharp}(w), & n \geq 0, \\
w^{n}\left[\Omega_{\sharp}(w)-\triangleleft_{-n-1}^{\sharp}(w)\right], & n<0,\end{cases}
\end{aligned}
$$

where

$$
\begin{aligned}
& \Omega_{\sharp}(w)=\frac{1}{2 \pi i} \int_{\Gamma} \frac{g_{*}(\tau)}{X_{\sharp}^{+}(\tau)(\tau-w)} \mathrm{d} \tau, \quad w \in \mathcal{C} \backslash \Gamma, \\
& \triangleright_{n-1}^{\sharp}(w)=\sum_{j=0}^{n-1}\left[\frac{1}{2 \pi i} \int_{\Gamma} \frac{g_{*}(\tau)}{X_{\sharp}^{+}(\tau)} \tau^{n-1-j} \mathrm{~d} \tau\right] w^{j} \quad(n \geq 0)
\end{aligned}
$$

is a polynomial of degree not greater than $(n-1)$,

$$
\triangleleft_{-n-1}^{\sharp}(w)=\sum_{j=0}^{-n-1}\left[\frac{1}{2 \pi i} \int_{\Gamma} \frac{g_{*}(\tau)}{X_{\sharp}^{+}(\tau) \tau^{j+1}} \mathrm{~d} \tau\right] w^{j} \quad(n<0)
$$

is a polynomial of degree not greater than $(-n-1)$, which is just the Taylor expansion of order $(-n-1)$ of $\Omega_{\sharp}$ at $w=0$. 
Returning to the $z$-plane, by using Lemma 3.1 and Lemma 3.2, we can easily obtain the solutions of $P R_{m, n}$ problem (3.1), which are divided into three cases.

Case 1. $m+n+\kappa>-1$. By (3.10), (3.11), and (3.16), the general solution is

$$
\Phi(z)=\Phi_{*}\left(e^{\frac{2 i z}{a}}\right)=e^{-\frac{2 n i z}{a}} \Phi_{\sharp}\left(e^{\frac{2 i z}{a}}\right), \quad z \in \mathcal{C} \backslash L,
$$

i.e.,

$$
\begin{aligned}
\Phi(z) & =X(z)\left[\Psi(z)+e^{-\frac{2 n i z}{a}} P_{m+n+\kappa}\left(e^{\frac{i z}{a}}\right)\right] \quad\left(P_{r} \in \Pi_{r}\right) \\
& =X(z)\left[\Psi(z)+e^{-\frac{2 n i z}{a}} T_{m+n+\kappa}(z)\right], \quad z \in \mathcal{C} \backslash L,
\end{aligned}
$$

where

$$
X(z)=X_{\sharp}\left(e^{\frac{2 i z}{a}}\right)= \begin{cases}e^{\Gamma(z)}, & z \in S^{+}, \\ \left(e^{\frac{2 i z}{a}}-w_{0}\right)^{-\kappa} e^{\Gamma(z)}, & z \in S^{-},\end{cases}
$$

in which

$$
\begin{aligned}
& \Gamma(z)=\Gamma_{\sharp}\left(e^{\frac{2 i z}{a}}\right) \quad\left(\Gamma_{\sharp} \text { is given in }(3.18)\right), \\
& \Psi(z)=e^{-\frac{2 n i z}{a}} \Psi_{\sharp}\left(e^{\frac{2 i z}{a}}\right) \quad\left(\Psi_{\sharp} \text { is given in }(3.19)\right), \\
& T_{m+n+\kappa}(z)=\sum_{j=0}^{m+n+\kappa} c_{j}\left[\cos \frac{2 j z}{a}+i \sin \frac{2 j z}{a}\right],
\end{aligned}
$$

is a trigonometric polynomial of degree not greater than $(m+n+\kappa)$ with arbitrary complex constants $c_{j}$, which shows that the general solution of $P R_{m, n}$ problem (3.1) has the degree of freedom $(m+n+\kappa+1)[14-16]$.

More specifically, $\Gamma$ and $\Psi$ are as follows by further calculations:

$$
\begin{aligned}
& \Gamma(z)=\frac{1}{a \pi} \int_{L_{0}} \log \left[\left(e^{\frac{2 i t}{a}}-w_{0}\right)^{-\kappa} G(t)\right] \frac{e^{\frac{2 i t}{a}}}{e^{\frac{2 i t}{a}}-e^{\frac{2 i z}{a}}} \mathrm{~d} t-C \quad \text { (by (3.30) and (3.5)) } \\
& =\frac{1}{2 a \pi} \int_{L_{0}} \log \left[\left(e^{\frac{2 i t}{a}}-w_{0}\right)^{-\kappa} G(t)\right]\left[\frac{e^{\frac{2 i t}{a}}+e^{\frac{2 i z}{a}}}{e^{\frac{2 i t}{a}}-e^{\frac{2 i z}{a}}}+1\right] \mathrm{d} t-C \\
& =\frac{1}{2 a \pi i} \int_{L_{0}} \log \left[\left(e^{\frac{2 i t}{a}}-w_{0}\right)^{-\kappa} G(t)\right] \cot \frac{t-z}{a} \mathrm{~d} t
\end{aligned}
$$

where we let

$$
\begin{aligned}
& C=\frac{1}{2 a \pi} \int_{L_{0}} \log \left[\left(e^{\frac{2 i t}{a}}-w_{0}\right)^{-\kappa} G(t)\right] \mathrm{d} t \\
& \begin{aligned}
\Psi(z) & =\frac{e^{-\frac{2 n i z}{a}}}{a \pi} \int_{L_{0}} \frac{\left[e^{\frac{2 n i t}{a}} g(t)\right] e^{\frac{2 i t}{a}}}{X^{+}(t)\left(e^{\frac{2 i t}{a}}-e^{\frac{2 i z}{a}}\right)} \mathrm{d} t \quad(\text { by }(3.19) \text { and }(3.31)) \\
& =\frac{e^{-\frac{2 n i z}{a}}}{2 a \pi} \int_{L_{0}} \frac{g(t)}{e^{-\frac{2 n i t}{a}} X^{+}(t)} \frac{e^{\frac{2 i t}{a}}+e^{\frac{2 i z}{a}}}{e^{\frac{2 i t}{a}}-e^{\frac{2 i z}{a}}} \mathrm{~d} t+\frac{e^{-\frac{2 n i z}{a}}}{2 a \pi} \int_{L_{0}} \frac{g(t)}{e^{-\frac{2 n i t}{a}} X^{+}(t)} \mathrm{d} t \\
& \equiv \frac{e^{-\frac{2 n i z}{a}}}{2 a \pi i} \int_{L_{0}} \frac{g(t)}{e^{-\frac{2 n i t}{a}} X^{+}(t)} \cot \frac{t-z}{a} \mathrm{~d} t+e^{-\frac{2 n i z}{a}} C^{*} \quad\left(C^{*} \text { is a constant }\right) .
\end{aligned}
\end{aligned}
$$


Case 2. $m+n+\kappa=-1$. By (3.10), (3.11) and (3.16), $P R_{m, n}$ problem (3.1) has the unique solution

$$
\Phi(z)=X(z) \Psi(z), \quad z \in \mathcal{C} \backslash L .
$$

Case 3. $m+n+\kappa<-1$. By (3.10), (3.11), (3.20), (3.21), and (3.22), $P R_{m, n}$ problem (3.1) has the unique solution (3.36) if and only if the following $-(m+n+\kappa+1)$ conditions are satisfied:

$$
\int_{L_{0}} \frac{g(t)}{X^{+}(t)} e^{\frac{2(n+j) i t}{a}} \mathrm{~d} t=0, \quad j=1, \ldots,-(m+n+\kappa+1),
$$

or

$$
\int_{L_{0}} \frac{g(t)}{e^{-\frac{2(n+1) i t}{a}} X^{+}(t)} P_{m+n+\kappa}\left(e^{\frac{2 i t}{a}}\right) \mathrm{d} t=0 \quad \text { for any } P_{m+n+\kappa} \in \Pi_{m+n+\kappa} .
$$

In this case, we also say the solution has the degree of freedom $(\kappa+m+n+1)$ (it is a negative integer!).

Based on the above discussion, we have the following.

Theorem 3.1 When $m+n+\kappa \geq 0$, the general solution of $P R_{m, n}$ is

$$
\Phi(z)=e^{-\frac{2 n i z}{a}} X(z)\left[\frac{1}{2 a \pi i} \int_{L_{0}} \frac{g(t)}{e^{-\frac{2 n i t}{a}} X^{+}(t)} \cot \frac{t-z}{a} \mathrm{~d} t+P_{m+n+\kappa}\left(e^{\frac{i z}{a}}\right)\right], \quad z \in \mathcal{C} \backslash L,
$$

where $P_{r}$ is an arbitrary polynomial of degree not greater than $r$. When $m+n+\kappa=-1$, it has the unique solution

$$
\Phi(z)=e^{-\frac{2 n i z}{a}} X(z) \frac{1}{2 a \pi i} \int_{L_{0}} \frac{g(t)}{e^{-\frac{2 n i t}{a}} X^{+}(t)}\left[i+\cot \frac{t-z}{a}\right] \mathrm{d} t, \quad z \in \mathcal{C} \backslash L .
$$

When $m+n+\kappa<-1$, it has the unique solution (3.40) if and only if (3.37) holds.

By recalling Remark 3.2, we also have

$$
\Psi(z)=\left\{\begin{array}{ll}
\Omega(z)+e^{-\frac{2 i n z}{a}} \triangleright_{n-1}(z), & n \geq 0, \\
\Omega(z)-\triangleleft_{-n-1}(z), & n<0,
\end{array} \quad z \in \mathcal{C} \backslash L,\right.
$$

where

$$
\begin{aligned}
\Omega(z) & =\Omega_{\sharp}\left(e^{\frac{2 i z}{a}}\right) \quad\left(\Omega_{\sharp} \text { is given in }(3.24)\right) \\
& =\frac{1}{a \pi} \int_{L_{0}} \frac{g(t) e^{\frac{2 i t}{a}}}{X^{+}(t)\left(e^{\frac{2 i t}{a}}-e^{\frac{2 i z}{a}}\right)} \mathrm{d} t \quad(\text { by }(3.7)) \\
& =\frac{1}{2 a \pi} \int_{L_{0}} \frac{g(t)}{X^{+}(t)} \frac{e^{\frac{2 i t}{a}}+e^{\frac{2 i z}{a}}}{e^{\frac{2 i t}{a}}-e^{\frac{2 i z}{a}}} \mathrm{~d} t+\frac{1}{2 a \pi} \int_{L_{0}} \frac{g(t)}{X^{+}(t)} \mathrm{d} t \\
& \equiv \frac{1}{2 a \pi i} \int_{L_{0}} \frac{g(t)}{X^{+}(t)} \cot \frac{t-z}{a} \mathrm{~d} t+C^{\prime} \quad\left(C^{\prime} \text { is a constant }\right),
\end{aligned}
$$




$$
\begin{aligned}
\triangleright_{n-1}(z)= & \triangleright_{n-1}^{\sharp}\left(e^{\frac{2 i z}{a}}\right) \quad\left(\triangleright_{n-1}^{\sharp}\right. \text { is given in (3.25)) } \\
= & \sum_{j=0}^{n-1}\left[\frac{1}{a \pi} \int_{L_{0}} \frac{g(t)}{X^{+}(t)} e^{\frac{2(n-j) i t}{a}} \mathrm{~d} t\right]\left[\cos \frac{2 j z}{a}+i \sin \frac{2 j z}{a}\right] \\
= & \sum_{j=0}^{n-1}\left\{\frac{1}{a \pi} \int_{L_{0}} \frac{g(t)}{X^{+}(t)}\left[\cos \frac{2(n-j) t}{a}+i \sin \frac{2(n-j) t}{a}\right] \mathrm{d} t\right\} \\
& \times\left[\cos \frac{2 j z}{a}+i \sin \frac{2 j z}{a}\right]
\end{aligned}
$$

is a trigonometric polynomial of degree not greater than $(n-1)$ (it vanishes while $n=0$ ),

$$
\begin{aligned}
\triangleleft_{-n-1}(z) & =\triangleleft_{-n-1}^{\sharp}\left(e^{\frac{2 i z}{a}}\right) \quad\left(P_{n-1}^{\sharp}\right. \text { is given in (3.26)) } \\
& =\sum_{j=0}^{-n-1}\left[\frac{1}{a \pi} \int_{L_{0}} \frac{g(t)}{X^{+}(t)} e^{-\frac{2 j i t}{a}} \mathrm{~d} t\right]\left[\cos \frac{2 j z}{a}+i \sin \frac{2 j z}{a}\right] \\
& =\sum_{j=0}^{-n-1}\left\{\frac{1}{a \pi} \int_{L_{0}} \frac{g(t)}{X^{+}(t)}\left[\cos \frac{2 j t}{a}-i \sin \frac{2 j t}{a}\right] \mathrm{d} t\right\}\left[\cos \frac{2 j z}{a}+i \sin \frac{2 j z}{a}\right]
\end{aligned}
$$

is a trigonometric polynomial of degree not greater than $(-n-1)$, which is just the Taylor expansion of order $(-n-1)$ of $\Omega(z)$ at $z=-\infty i$.

Remark 3.3 Case 1 may be divided into two subcases: (1) when $\kappa+m>-1$ and $n \geq 0$, the polynomial $\triangleright_{n-1}$ may be omitted and merged to $T_{m+n+\kappa}$; (2) when $\kappa+m \leq-1$ and $n>0$, the polynomial $\triangleright_{n-1}$ must be divided into two parts, in which the polynomial of degree not greater than $(m+n+\kappa)$ may be merged into $T_{m+n+\kappa}$ and the other part is still kept back. In addition, the constant $C^{\prime}$ in (3.42) may be omitted by merging it to $T_{m+n+\kappa}$.

We restate Theorem 3.1 as follows.

Theorem 3.2 The general solution of $P R_{m, n}$ problem (3.1) always has the degree of freedom $(m+n+\kappa+1)$. In detail, there are six cases.

(1) When $\kappa+m \geq 0$ and $n \geq 0$, the general solution of $P R_{m, n}$ problem (3.1) is

$$
\begin{aligned}
\Phi(z)= & X(z)\left\{\frac{1}{2 a \pi i} \int_{L_{0}} \frac{g(t)}{X^{+}(t)} \cot \frac{t-z}{a} \mathrm{~d} t\right. \\
& \left.+\sum_{j=0}^{m+n+\kappa} c_{j}\left[\cos \frac{2(j-n) z}{a}+i \sin \frac{2(j-n) z}{a}\right]\right\},
\end{aligned}
$$

where the $c_{j}$ are arbitrary complex constants.

(2) When $n-1>\kappa+m+n \geq 0$, the general solution of $P R_{m, n}$ problem (3.1) is

$$
\begin{aligned}
\Phi(z)= & X(z)\left\{\frac{1}{2 a \pi i} \int_{L_{0}} \frac{g(t)}{X^{+}(t)} \cot \frac{t-z}{a} \mathrm{~d} t\right. \\
& +\sum_{j=0}^{m+n+\kappa} c_{j}\left[\cos \frac{2(j-n) z}{a}+i \sin \frac{2(j-n) z}{a}\right]
\end{aligned}
$$




$$
\begin{aligned}
& +\sum_{j=m+n+\kappa+1}^{n-1} \frac{1}{a \pi} \int_{L_{0}} \frac{g(t)}{X^{+}(t)}\left[\cos \frac{2(n-j) t}{a}+i \sin \frac{2(n-j) t}{a} \mathrm{~d} t\right] \\
& \left.\times\left[\cos \frac{2 j z}{a}+i \sin \frac{2 j z}{a}\right]\right\}
\end{aligned}
$$

where the $c_{j}$ are arbitrary complex constants.

(3) When $\kappa+m+n \geq 0$ and $n<0$, the general solution of $P R_{m, n}$ problem (3.1) is

$$
\begin{aligned}
\Phi(z)= & X(z)\left\{\frac{1}{2 a \pi i} \int_{L_{0}} \frac{g(t)}{X^{+}(t)} \cot \frac{t-z}{a} \mathrm{~d} t+\sum_{j=0}^{m+n+\kappa} c_{j}\left[\cos \frac{2(j-n) z}{a}+i \sin \frac{2(j-n) z}{a}\right]\right. \\
& \left.-\sum_{j=0}^{-n-1} \frac{1}{a \pi} \int_{L_{0}} \frac{g(t)}{X^{+}(t)}\left[\cos \frac{2 j t}{a}-i \sin \frac{2 j t}{a}\right] \mathrm{d} t\left[\cos \frac{2 j z}{a}+i \sin \frac{2 j z}{a}\right]\right\}
\end{aligned}
$$

where the $c_{j}$ are arbitrary complex constants.

(4) When $\kappa+m+n=-1$ and $n \geq 0$, the unique solution of $P R_{m, n}$ problem (3.1) is

$$
\begin{aligned}
\Phi(z)= & X(z)\left\{\frac{1}{2 a \pi i} \int_{L_{0}} \frac{g(t)}{X^{+}(t)} \cot \frac{t-z}{a} \mathrm{~d} t+\frac{1}{2 a \pi} \int_{L_{0}} \frac{g(t)}{X^{+}(t)} \mathrm{d} t\right. \\
& +\sum_{j=0}^{n-1} \frac{1}{a \pi} \int_{L_{0}} \frac{g(t)}{X^{+}(t)}\left[\cos \frac{2(n-j) t}{a}+i \sin \frac{2(n-j) t}{a} \mathrm{~d} t\right] \\
& \left.\times\left[\cos \frac{2 j z}{a}+i \sin \frac{2 j z}{a}\right]\right\},
\end{aligned}
$$

where $\sum \equiv 0$, while $n=0$.

(5) When $\kappa+m+n=-1$ and $n<0$, the unique solution of $P R_{m, n}$ problem (3.1) is

$$
\begin{aligned}
\Phi(z)= & X(z)\left\{\frac{1}{2 a \pi i} \int_{L_{0}} \frac{g(t)}{X^{+}(t)} \cot \frac{t-z}{a} \mathrm{~d} t+\frac{1}{2 a \pi} \int_{L_{0}} \frac{g(t)}{X^{+}(t)} \mathrm{d} t\right. \\
& \left.-\sum_{j=0}^{-n-1} \frac{1}{a \pi} \int_{L_{0}} \frac{g(t)}{X^{+}(t)}\left[\cos \frac{2 j t}{a}-i \sin \frac{2 j t}{a}\right] \mathrm{d} t\left[\cos \frac{2 j z}{a}+i \sin \frac{2 j z}{a}\right]\right\} .
\end{aligned}
$$

(6) When $\kappa+m+n<-1$, the unique solution of $P R_{m, n}$ problem (3.1) is

$$
\Phi(z)=\left\{\begin{aligned}
X(z)\{ & \frac{1}{2 a \pi i} \int_{L_{0}} \frac{g(t)}{X^{+}(t)} \cot \frac{t-z}{a} \mathrm{~d} t+\frac{1}{2 a \pi} \int_{L_{0}} \frac{g(t)}{X^{+}(t)} \mathrm{d} t \\
& +\sum_{j=0}^{n-1} \frac{1}{a \pi} \int_{L_{0}} \frac{g(t)}{X^{+}(t)}\left[\cos \frac{2(n-j) t}{a}+i \sin \frac{2(n-j) t}{a} \mathrm{~d} t\right] \\
& \left.\times\left[\cos \frac{2 j z}{a}+i \sin \frac{2 j z}{a}\right]\right\}, \quad n \geq 0, \\
X(z) & \left\{\frac{1}{2 a \pi i} \int_{L_{0}} \frac{g(t)}{X^{+}(t)} \cot \frac{t-z}{a} \mathrm{~d} t+\frac{1}{2 a \pi} \int_{L_{0}} \frac{g(t)}{X^{+}(t)} \mathrm{d} t\right. \\
& -\sum_{j=0}^{-n-1} \frac{1}{a \pi} \int_{L_{0}} \frac{g(t)}{X^{+}(t)}\left[\cos \frac{2 j t}{a}-i \sin \frac{2 j t}{a}\right] \mathrm{d} t \\
& \left.\times\left[\cos \frac{2 j z}{a}+i \sin \frac{2 j z}{a}\right]\right\}, \quad n<0,
\end{aligned}\right.
$$


if and only if the $-(\kappa+m+n+1)$ conditions

$$
\int_{L_{0}} \frac{g(t)}{X^{+}(t)}\left[\cos \frac{2 j t}{a}+i \sin \frac{2 j t}{a}\right] \mathrm{d} t=0, \quad j=n+1, \ldots,-(m+\kappa+1)
$$

are satisfied.

\subsection{Relative discussions}

Let

$$
X_{0}(z)= \begin{cases}e^{\Gamma_{0}(z)}, & z \in S^{+} \\ {\left[\tan \frac{z}{a}\right]^{-\kappa} e^{\Gamma_{0}(z)},} & z \in S^{-}\end{cases}
$$

where

$$
\Gamma_{0}(z)=\frac{1}{2 a \pi \mathrm{i}} \int_{L_{0}} \log \left[\tan ^{-\kappa} \frac{t}{a} G(t)\right] \cot \frac{t-z}{a} \mathrm{~d} t
$$

Then it is easy to see, from (3.33),

$$
\begin{aligned}
\Gamma(z)-\Gamma_{0}(z) & =\frac{1}{2 a \pi i} \int_{L_{0}} \log \left[\frac{e^{\frac{2 i t}{a}}-w_{0}}{\tan \frac{t}{a}}\right]^{-\kappa} \cot \frac{t-z}{a} \mathrm{~d} t \\
& = \begin{cases}\log \left[\frac{e^{\frac{2 i z}{a}}-1}{\tan \frac{z}{a}}\right]^{-\kappa}, & z \in S^{+}, \\
0, & z \in S^{-} .\end{cases}
\end{aligned}
$$

Thus,

$$
\begin{aligned}
& X(z)=i^{-\kappa}\left[1+e^{\frac{2 i z}{a}}\right]^{-\kappa} X_{0}(z), \quad z \in \mathcal{C} \backslash L, \\
& X^{ \pm}(t)=i^{-\kappa}\left[1+e^{\frac{2 i t}{a}}\right]^{-\kappa} X_{0}^{ \pm}(t), \quad t \in L .
\end{aligned}
$$

Then

$$
\begin{aligned}
e^{-\frac{2 n i z}{a}} X(z) & =i^{-\kappa} e^{-\frac{2 n i z}{a}}\left[1+e^{\frac{2 i z}{a}}\right]^{-\kappa} X_{0}(z)\left[i+\tan \frac{z}{a}\right]^{-m-n-\kappa}\left[i+\tan \frac{z}{a}\right]^{m+n+\kappa} \\
& =(-2)^{-\kappa}[2 i]^{-m-n} e^{-\frac{2 n i z}{a}}\left[1+e^{\frac{2 i z}{a}}\right]^{m+n} X_{0}(z)\left(i+\tan \frac{z}{a}\right)^{m+n+\kappa} \\
& =(-2)^{-m-n-\kappa}[i]^{m+n}\left[1+e^{\frac{2 i z}{a}}\right]^{m}\left[1+e^{-\frac{2 i z}{a}}\right]^{n} X_{0}(z)\left(i+\tan \frac{z}{a}\right)^{m+n+\kappa} \\
& =\Upsilon(z) \Delta^{m+n+\kappa}(z),
\end{aligned}
$$

where

$$
\begin{aligned}
& \Upsilon(z)=(-2)^{-\kappa}[2 i]^{-m-n}\left[1+e^{\frac{2 i z}{a}}\right]^{m}\left[1+e^{-\frac{2 i z}{a}}\right]^{n} X_{0}(z), \\
& \Delta(z)=i+\tan \frac{z}{a} .
\end{aligned}
$$


Then

$$
e^{-\frac{2 n i z}{a}} X(z)\left(e^{\frac{2 i z}{a}}\right)^{j}=\Upsilon(z)\left(i-\tan \frac{z}{a}\right)^{j}\left(i+\tan \frac{z}{a}\right)^{m+n+\kappa-j} .
$$

So,

$$
e^{-\frac{2 n i z}{a}} X(z) P_{m+n+\kappa}\left(\frac{2 i z}{a}\right)=\Upsilon(z) P_{m+n+\kappa}^{*}\left(\tan \frac{z}{a}\right) \quad\left(P_{r}^{*} \in \Pi_{r}\right) .
$$

Noting

$$
e^{\frac{i z}{a}} \Delta(z)=\frac{i}{\cos \frac{z}{a}}, \quad e^{\frac{2 i t}{a}}-e^{\frac{2 i z}{a}}=\frac{2 i}{\Delta(t) \Delta(z)}\left[\tan \frac{z}{a}-\tan \frac{t}{a}\right],
$$

which result in

$$
\frac{e^{\frac{2 i t}{a}}}{e^{\frac{2 i t}{a}}-e^{\frac{2 i z}{a}}}=\frac{1}{2 i} \frac{\triangle(z)}{\triangle(t)} \frac{1}{\left[\tan \frac{t}{a}-\tan \frac{z}{a}\right] \cos ^{2} \frac{t}{a}},
$$

we have

$$
\begin{aligned}
& X(z) \Psi(z) \\
& =\frac{e^{-\frac{2 n i z}{a}} X(z)}{a \pi} \int_{L_{0}} \frac{g(t) e^{\frac{2 i t}{a}}}{e^{\frac{-2 n i t}{a}} X^{+}(t)\left(e^{\frac{2 i t}{a}}-e^{\frac{2 i z}{a}}\right)} \mathrm{d} t \quad \text { (by the first equality of (3.35)) } \\
& =\frac{\Upsilon(z)}{2 a \pi i} \int_{L_{0}} \frac{\Delta^{m+n+\kappa+1}(z)}{\Delta^{m+n+\kappa+1}(t)} \frac{g(t)}{\Upsilon^{+}(t)\left[\tan \frac{t}{a}-\tan \frac{z}{a}\right] \cos ^{2} \frac{t}{a}} \mathrm{~d} t \quad \text { (by (3.63)) } \\
& =\frac{\Upsilon(z)}{2 a \pi i} \int_{L_{0}} \frac{\Delta^{m+n+\kappa+1}(z)}{\Delta^{m+n+\kappa+1}(t)} \frac{g(t)}{\Upsilon^{+}(t)}\left[\cot \frac{t-z}{a}+\tan \frac{t}{a}\right] \mathrm{d} t .
\end{aligned}
$$

We will discuss three cases.

Case 1. When $m+n+\kappa \geq 0$, we get

$$
\begin{aligned}
X(z) \Psi(z) & =\Upsilon(z)\left[P_{m+n+\kappa}^{\star}\left(\tan \frac{z}{a}\right)+\Psi_{0}(z)\right] \quad\left(P_{r}^{\star} \in \Pi_{r}\right) \\
& =\Upsilon(z)\left[P_{m+n+\kappa}^{\sharp}\left(\tan \frac{z}{a}\right)+\frac{1}{2 a \pi i} \int_{L_{0}} \frac{g(t)}{\Upsilon^{+}(t)} \cot \frac{t-z}{a} \mathrm{~d} t\right] \quad\left(P_{r}^{\sharp} \in \Pi_{r}\right),
\end{aligned}
$$

where

$$
\Psi_{0}(z)=\frac{1}{2 a \pi i} \int_{L_{0}} \frac{g(t)}{\Upsilon^{+}(t)} \cot \frac{t-z}{a} \mathrm{~d} t+\frac{1}{2 a \pi} \int_{L_{0}} \frac{g(t)}{\Upsilon^{+}(t)} \tan \frac{t}{a} \mathrm{~d} t
$$

Case 2. When $m+n+\kappa=-1$, we get

$$
X(z) \Psi(z)=\frac{\Upsilon(z)}{2 a \pi i} \int_{L_{0}} \frac{g(t)}{\Upsilon^{+}(t)}\left[\cot \frac{t-z}{a}+\tan \frac{t}{a}\right] \mathrm{d} t=\Upsilon(z) \Psi_{0}(z)
$$


Case 3. When $m+n+\kappa<-1$, we get

$$
\begin{aligned}
& X(z) \Psi(z)=\frac{\Upsilon(z)}{2 a \pi i} \int_{L_{0}} \frac{\Delta^{-(m+n+\kappa+1)}(t)}{\Delta^{-(m+n+\kappa+1)}(z)} \frac{g(t)}{\Upsilon^{+}(t)}\left[\cot \frac{t-z}{a}+\tan \frac{t}{a}\right] \mathrm{d} t \quad(\text { by }(3.64)) \\
& =\Upsilon(z)\left[\Psi_{0}(z)+\frac{1}{2 a \pi i \Delta^{-(m+n+\kappa+1)}(z)} \Theta(z)\right] \text {, }
\end{aligned}
$$

where

$$
\begin{aligned}
\Theta(z) & =\int_{L_{0}}\left[\Delta^{-(m+n+\kappa+1)}(t)-\Delta^{-(m+n+\kappa+1)}(z)\right] \frac{g(t)}{\Upsilon^{+}(t)}\left[\cot \frac{t-z}{a}+\tan \frac{t}{a}\right] \mathrm{d} t \\
& =\int_{L_{0}} \frac{g(t)}{\Upsilon^{+}(t) \cos ^{2} \frac{t}{a}} \sum_{j=0}^{-(m+n+\kappa+2)} \Delta^{j}\left(\tan \frac{t}{a}\right) \Delta^{-(m+n+\kappa+2)-j}\left(\tan \frac{z}{a}\right) \mathrm{d} t \\
& =\sum_{j=0}^{-(m+n+\kappa+2)}\left[\int_{L_{0}} \frac{g(t)}{\Upsilon^{+}(t) \cos ^{2} \frac{t}{a}}\left(i+\tan \frac{t}{a}\right)^{j} \mathrm{~d} t\right] \Delta^{-(m+n+\kappa+2)-j}\left(\tan \frac{z}{a}\right) .
\end{aligned}
$$

We note that

$$
\begin{aligned}
& \int_{L_{0}} \frac{g(t)}{e^{\frac{-2 n i t}{a}} X^{+}(t)} e^{\frac{2 i t}{a}} P_{-(m+n+\kappa)-2}\left(e^{\frac{2 i t}{a}}\right) \mathrm{d} t \quad\left(P_{r} \in \Pi_{r}\right) \\
& \quad=\int_{L_{0}} \frac{g(t)}{\Upsilon(t)} \frac{1}{\Delta^{m+n+\kappa+2}(t)} P_{-(m+n+\kappa)-2}\left(e^{\frac{2 i t}{a}}\right)\left[e^{\frac{2 i t}{a}} \Delta^{2}(t)\right] \mathrm{d} t \\
& =\int_{L_{0}} \frac{g(t)}{\Upsilon(t) \cos ^{2} \frac{t}{a}} \frac{1}{\Delta^{m+n+\kappa+2}(t)} P_{-(m+n+\kappa)-2}^{\natural}\left(\frac{i-\tan \frac{t}{a}}{i+\tan \frac{t}{a}}\right) \mathrm{d} t \quad\left(P_{r}^{\natural} \in \Pi_{r}\right) \\
& =\int_{L_{0}} \frac{g(t)}{\Upsilon(t) \cos ^{2} \frac{t}{a}} P_{-(m+n+\kappa)-2}^{\mathbb{I}}\left(\tan \frac{t}{a}\right) \mathrm{d} t \quad\left(P_{r}^{\mathbb{I}} \in \Pi_{r}\right) .
\end{aligned}
$$

To the contrary, we easily see that

$$
\begin{aligned}
\int_{L_{0}} & \frac{g(t)}{\Upsilon(t) \cos ^{2} \frac{t}{a}} P_{-(m+n+\kappa)-2}^{\mathbb{I}}\left(\tan \frac{t}{a}\right) \mathrm{d} t \quad\left(P_{r}^{\mathbb{I}} \in \Pi_{r}\right) \\
= & \int_{L_{0}} \frac{g(t)}{\Upsilon(t)}\left[e^{\frac{2 i t}{a}} \Delta^{2}(t)\right] P_{-(m+n+\kappa)-2}^{\sharp}\left(\frac{e^{\frac{2 i t}{a}}-1}{i\left[e^{\frac{2 i t}{a}}+1\right]}\right) \mathrm{d} t \quad\left(P_{r}^{\sharp} \in \Pi_{r}\right) \\
= & \int_{L_{0}} \frac{g(t)}{\Upsilon(t)}\left[e^{\frac{2 i t}{a}} \Delta^{2}(t)\right]\left[1+e^{\frac{2 i t}{a}}\right]^{m+n+\kappa+2} P_{-(m+n+\kappa+2)}\left(e^{\frac{2 i t}{a}}\right) \mathrm{d} t \quad\left(P_{r}^{*} \in \Pi_{r}^{*}\right) \\
= & \int_{L_{0}} \frac{g(t) e^{\frac{2 i t}{a}}}{X^{+}(t)} P_{-(m+n+\kappa+2)}\left(e^{\frac{2 i t}{a}}\right) \mathrm{d} t \quad\left(P_{r} \in \Pi_{r}\right) .
\end{aligned}
$$

So, from (3.70) and (3.71), we know that (3.51) is equivalent to

$$
\int_{L_{0}} \frac{g(t)}{\Upsilon(t) \cos ^{2} \frac{t}{a}}\left(\tan \frac{t}{a}\right)^{j} \mathrm{~d} t=0, \quad j=0,1, \ldots,-(m+n+\kappa+2) .
$$

Also, by (3.68) and (3.69), we have (3.67) when one of (3.51) and (3.72) is satisfied. 
Noting that, from (3.58),

$$
\Upsilon(z)=C^{\sharp} \cos ^{m+n} \frac{z}{a} e^{\frac{(m-n) i z}{a}},
$$

where

$$
C^{\sharp}=(-2)^{-\kappa}(-i)^{m+n}
$$

is a constant, we may restate Theorem 3.1 in another form.

Theorem 3.3 When $m+n+\kappa \geq 0$, the general solution of $P R_{m, n}$ is

$$
\begin{aligned}
\Phi(z)= & \cos ^{m+n} \frac{z}{a} e^{\frac{(m-n) i z}{a}} \\
& \times\left[P_{m+n+\kappa}^{\sharp}\left(\tan \frac{z}{a}\right)+\frac{1}{2 a \pi i} \int_{L_{0}} \frac{g(t)}{\cos ^{m+n} \frac{t}{a} e^{\frac{(m-n) i t}{a}} X_{0}^{+}(t)} \cot \frac{t-z}{a} \mathrm{~d} t\right],
\end{aligned}
$$

where $P_{r}^{\sharp}$ is an arbitrary polynomial of degree not greater than $r$. When $m+n+\kappa=-1$, it has the unique solution

$$
\Phi(z)=\frac{\cos ^{m+n} \frac{z}{a} e^{\frac{(m-n) i z}{a}}}{2 a \pi i} \int_{L_{0}} \frac{g(t)}{\cos ^{m+n} \frac{t}{a} e^{\frac{(m-n) i t}{a}} X_{0}(t)}\left[\cot \frac{t-z}{a}+\tan \frac{t}{a}\right] \mathrm{d} t .
$$

When $m+n+\kappa<-1$, it has the unique solution (3.76) if and only if

$$
\int_{L_{0}} \frac{g(t)}{\cos ^{m+n} \frac{t}{a} e^{\frac{(m-n) i t}{a}} X_{0}^{+}(t)}\left[\tan \frac{t}{a}\right]^{j} \mathrm{~d} t=0, \quad j=0,1, \ldots,-(m+n+\kappa+2),
$$

hold.

Now, we immediately see that Theorem 3.3 generalizes some previous results. The method used here is more straightforward than that used to obtain the previous results.

Example 3.1 When $m=n \geq 0$ and $L$ is the periodic closed contour, the result of [17] is easily reobtained from Theorem 3.3 and (3.77). In particular, when $m=n=0$ we reobtain the results of $[7,9]$. These researchers just used the tangent conformal mapping

$$
w=\tan \frac{z}{a}, \quad z \in \mathcal{C} \backslash L .
$$

Example 3.2 When $L$ is the real axis, we generalize the result of [17], in which the authors only discussed the case of $m=n \geq 0$.

\section{Periodic Hilbert boundary value problems}

In this section, we discuss the periodic Hilbert boundary value problems ( $\mathrm{PH}$ problems). We expect to obtain concrete expressions for their solutions as well as the conditions of solvability. Two kinds of $\mathrm{PH}$ problems are to solved in detail, which are, respectively, the $\mathrm{PH}$ problem on the circumferences and the $\mathrm{PH}$ problem on the real axis. The two kinds of $\mathrm{PH}$ problems are also of special importance in practice. 


\section{1 $\mathrm{PH}$ problem on the real axis}

Let $\mathcal{Z}^{+}$and $\mathcal{Z}^{-}$represent, respectively, the upper and lower half-plane. Denote the real axis by $\mathcal{X}$. We consider the following Hilbert boundary problem.

$P H_{n}$ problem: Find a periodic holomorphic function $\Phi(z)$ in the upper half-plane $\mathcal{Z}^{+}$ and continuous on $\overline{\mathcal{Z}^{+}}\left(\mathcal{Z}^{+} \cup \mathcal{X}\right)$, with period $a \pi$, such that

$$
\left\{\begin{array}{l}
\operatorname{Re}\left\{[a(t)+i b(t)] \Phi^{+}(t)\right\}=c(t), \quad t \in \mathcal{X}, \\
\operatorname{eOrd}[\Phi](+\infty i) \leq n
\end{array}\right.
$$

where the input functions $a(t), b(t), c(t)$ are periodic real-valued functions with period $a \pi$ on the $\mathcal{X}$-axis and $a, b, c \in H(\mathcal{X})$, and

$$
|a(t)+b(t)|=1, \quad t \in \mathcal{X} \text { (normalized condition). }
$$

In [7], Lu discussed the $P H_{0}$ problem. He transferred $P H_{0}$ to an ordinary Hilbert boundary value problem in the upper half $w$-plane by using the tangent type conformal mapping (3.78), which seems to be a bit complicated. So we prefer to transform directly a $P H_{0}$ problem into a $P R_{n, n}$ problem by using the reflection method based on the principle of the so-called symmetric extension [18]. For the sake of convenience, it is necessary to recall here some basic processes of the reflection method $[7,18]$.

For a function $\Phi(z)$ in $\mathcal{Z}^{+}$, we define a function in $\mathcal{Z}^{-}$by

$$
\bar{\Phi}(z)=\overline{\Phi(\bar{z})},
$$

which is called the accompanying function of $\Phi$. Then

$$
(\mathcal{E}[\Phi])(z)= \begin{cases}\Phi(z), & \text { when } z \in \mathcal{Z}^{+}, \\ \Phi(z), & \text { when } z \in \mathcal{Z}^{-},\end{cases}
$$

is called symmetric extension or symmetric function of $\Phi$.

Similarly, if the original function $\Phi(z)$ is defined in $\mathcal{Z}^{-}$, then its accompanying function $\bar{\Phi}(z)$ determined by (4.3) is defined in $\mathcal{Z}^{+}$. Then

$$
(\mathcal{E}[\Phi])(z)= \begin{cases}\bar{\Phi}(z), & \text { when } z \in \mathcal{Z}^{+} \\ \Phi(z), & \text { when } z \in \mathcal{Z}^{-}\end{cases}
$$

Moreover, we have

$$
\overline{\bar{\Phi}}(z)=\Phi(z), \quad z \in \mathcal{Z}^{+}\left(z \in \mathcal{Z}^{-}\right) .
$$

If $\mho$ is defined on $\mathcal{Z}^{+} \cup \mathcal{Z}^{-}$, say,

$$
\mho(z)= \begin{cases}\mho^{+}(z), & z \in \mathcal{Z}^{+} \\ \mho^{-}(z), & z \in \mathcal{Z}^{-}\end{cases}
$$

then

$$
\mho_{\dagger}(z)= \begin{cases}\overline{\mho^{-}}(z), & z \in \mathcal{Z}^{+}, \\ \overline{\mho^{+}}(z), & z \in \mathcal{Z}^{-},\end{cases}
$$


is called the reflective function of $\mho$. Obviously, by (4.6)

$$
\mho(z)=\left(\mho_{\dagger}\right) \dagger(z) .
$$

In particular, if

$$
\mho_{\dagger}(z)=\mho(z)
$$

then we say that $\mho$ is a self-reflection function. Obviously, by (4.9),

$$
(\mathscr{R}[\mho])(z)=\frac{\mho(z)+\mho_{\dagger}(z)}{2}
$$

is a self-reflection function, which is called the self-reflection function of $\mho$. For the sake of convenience, we call the above steps from $\Phi$ to $\mathscr{R}[\mathcal{E}[\Phi]]$ the self-reflex action of $\Phi$.

\section{Example 4.1}

$$
\left(e^{i z}\right)_{\dagger}=e^{-i z}, \quad\left(e^{-i z}\right)_{\dagger}=e^{i z}, \quad(\cot z)_{\dagger}=\cot z .
$$

Now it is easy to see that, if $\Phi$ is a periodic holomorphic function in the upper half-plane $\mathcal{Z}^{+}$and continuous on $\overline{\mathcal{Z}^{+}}$, with period $a \pi$, then $\bar{\Phi}(z)$ is a periodic holomorphic function in the lower half-plane $\mathcal{Z}^{-}$and continuous on $\overline{\mathcal{Z}^{-}}\left(\mathcal{Z}^{-} \cup \mathcal{X}\right)$, with period $a \pi$, and

$$
\begin{aligned}
& \overline{\Phi^{+}(t)}=(\bar{\Phi})^{-}(t), \quad t \in \mathcal{X}, \\
& \operatorname{eOrd}[\Phi](+\infty i)=\operatorname{eOrd}\left[\Phi_{\dagger}\right](-\infty i) .
\end{aligned}
$$

In brief, if $\Phi$ is the solution of $P H_{n}$ problem (4.1), then, noting that all input functions $a$, $b, c$ are real-valued, both its symmetric extension $\mathcal{E}[\Phi]$ given by (4.4) and its reflection function $(\mathcal{E}[\Phi])_{\uparrow}$ given by $(4.8)$ are the solution of the following $P R_{n, n}$ problem:

$$
\left\{\begin{array}{l}
\nabla^{+}(t)=-\frac{a(t)-i b(t)}{a(t)+i b(t)} \nabla^{-}(t)+\frac{2 c(t)}{a(t)+i b(t)}, \quad t \in \mathcal{X} \\
\operatorname{eOrd}[\nabla](+\infty i)=\operatorname{eOrd}[\nabla](-\infty i)=n
\end{array}\right.
$$

So is the self-reflection function $\mathscr{R}[\mathcal{E}[\Phi]]$ given by (4.11). Thus, we have the following lemma, that is, the symmetric extension principle.

Lemma 4.1 The general solution of $P H_{n}$ problem (4.1) should be

$$
\Phi(z)=(\mathscr{R}[\nabla])(z)=\frac{\nabla(z)+\nabla_{\ddagger}(z)}{2}=\frac{\nabla(z)+\bar{\nabla}(z)}{2}, \quad z \in \mathcal{Z}^{+},
$$

where $\nabla$ is the solution of the $P R_{n, n}$ problem (4.15).

Let

$$
\kappa=\frac{1}{\pi}[\arg [a(t)-i b(t)]]_{-\frac{a \pi}{2}}^{\frac{a \pi}{2}}=: 2 \ell,
$$

which is an even number. 
When $n+\ell \geq 0$, from (3.39) and (3.3), the general solution of the $P R_{n, n}$ problem (4.15) is

$$
\begin{aligned}
\nabla(z) & =e^{-\frac{2 n i z}{a}} X(z)\left[\frac{1}{a \pi i} \int_{-\frac{a \pi}{2}}^{\frac{a \pi}{2}} \frac{e^{\frac{2 n i t}{a}} c(t)}{X^{+}(t)[a(t)+i b(t)]} \cot \frac{t-z}{a} \mathrm{~d} t+P_{2(n+\ell)}\left(e^{\frac{i z}{a}}\right)\right] \\
z & \in \mathcal{C} \backslash \mathcal{X},
\end{aligned}
$$

where

$$
P_{2(\ell+n)}(z)=\sum_{j=0}^{2(n+\ell)} c_{j} z^{j}
$$

with $(2 n+\kappa+1)$ free constants $c_{j}$,

$$
X(z)= \begin{cases}e^{\Gamma(z)}, & z \in \mathcal{Z}^{+}, \\ e^{\frac{-2 \kappa i z}{a}} e^{\Gamma(z)}, & z \in \mathcal{Z}^{-},\end{cases}
$$

where

$$
\Gamma(z)=\frac{1}{a \pi} \int_{-\frac{a \pi}{2}}^{\frac{a \pi}{2}} \Theta(t) \cot \frac{t-z}{a} \mathrm{~d} t, \quad z \in \mathcal{C} \backslash \mathcal{X},
$$

where

$$
\Theta(t)=-\frac{\kappa t}{a}+\arg [a(t)-i b(t)]+\frac{\pi}{2}
$$

is a real-valued function.

Noting (4.12), we have

$$
\left\{\begin{array}{l}
\Gamma_{\dagger}(z)=\Gamma(z), \quad X_{\dagger}(z)=e^{\frac{2 \kappa i z}{a}} X(z), \quad z \in \mathcal{C} \backslash \mathcal{X}, \\
\overline{\Gamma^{+}(t)}=-2 i \Theta(t)+\Gamma^{+}(t), \\
\Gamma^{+}(t)=i \Theta(t)+\frac{1}{a \pi} \int_{-a \pi}^{+a \pi} \Theta(\tau) \cot \frac{\tau-t}{a} \mathrm{~d} \tau, \quad t \in \mathcal{X}, \\
\overline{X^{+}(t)}=-e^{\frac{2 \kappa i t}{a}}[a(t)+i b(t)]^{2} X^{+}(t), \\
X^{+}(t)=-e^{-\frac{2 \kappa i t}{a}}[a(t)-i b(t)]^{2} \bar{X}^{+}(t), \quad t \in \mathcal{X},
\end{array}\right.
$$

and

$$
\left[P_{2(n+\ell)}\left(e^{\frac{i z}{a}}\right)\right]_{\dagger}=\sum_{j=0}^{2(\ell+n)} \overline{c_{j}} e^{\frac{-j i z}{a}}
$$

From (4.16), (4.18), (4.23), and Theorem 3.1, we get the solution of $P H_{n}$ problem (4.1)

$$
\begin{aligned}
\Phi(z)= & X(z)\left[\frac{e^{-\frac{2 n i z}{a}}}{2 a \pi i} \int_{-\frac{a \pi}{2}}^{\frac{a \pi}{2}} \frac{c(t)}{e^{-\frac{2 n i t}{a}} X^{+}(t)[a(t)+i b(t)]} \cot \frac{t-z}{a} \mathrm{~d} t+T_{2(n+\ell)}(z)\right. \\
& \left.+\frac{e^{\frac{2(n+\kappa) i z}{a}}}{2 a \pi i} \int_{-\frac{a \pi}{2}}^{\frac{a \pi}{2}} \frac{c(t)}{e^{\frac{2(n+\kappa) i t}{a}} X^{+}(t)[a(t)+i b(t)]} \cot \frac{t-z}{a} \mathrm{~d} t\right], \quad z \in \mathcal{Z}^{+},
\end{aligned}
$$


where

$$
T_{2(n+\ell)}(z)=a_{0}+\sum_{j=1}^{2(n+\ell)} z\left[a_{j} \cos \frac{j z}{a}+i b_{j} \sin \frac{j z}{a}\right]
$$

is a trigonometric polynomial of degree not greater than $2(n+\ell)$ with $2(n+\ell)+1$ arbitrary real coefficients $a_{j}$ and $b_{j}$, denoted by $T_{2(n+\ell)} \in H_{2(n+\ell)}^{T}$.

When $n+\ell \leq-1$, from Theorem 3.1, the unique solution of $P R_{n, n}$ problem (4.15) is

$$
\Delta(z)=\frac{e^{-\frac{2 n i z}{a}} X(z)}{a \pi i} \int_{-\frac{a \pi}{2}}^{\frac{a \pi}{2}} \frac{c(t)}{e^{-\frac{2 n i t}{a}}[a(t)+i b(t)] X^{+}(t)}\left[i+\cot \frac{t-z}{a}\right] \mathrm{d} t, \quad z \in \mathcal{C} \backslash \mathcal{X},
$$

if and only if the $-(2 n+2 \ell+1)$ conditions

$$
\int_{-\frac{a \pi}{2}}^{\frac{a \pi}{2}} \frac{c(t)}{[a(t)+i b(t)] X^{+}(t)} e^{\frac{2 i j t}{a}} \mathrm{~d} t=0, \quad j=n+1, \ldots,-(n+2 \ell+1),
$$

are fulfilled.

Now we find the solution of $P H_{n}$ problem (4.1) by the self-reflex action. From (4.27), we get

$$
\begin{aligned}
\Delta_{\dagger}(z) & =\frac{e^{\frac{2(n+\kappa) i z}{a} X(z)}}{a \pi i} \int_{-\frac{a \pi}{2}}^{\frac{a \pi}{2}} \frac{c(t)}{e^{\frac{2(n+\kappa) i t}{a}}[a(t)+i b(t)] X^{+}(t)}\left[\cot \frac{t-z}{a}-i\right] \mathrm{d} t \\
& =\frac{e^{\frac{2(n+\kappa) i z}{a}} X(z)}{a \pi i} \int_{-\frac{a \pi}{2}}^{\frac{a \pi}{2}} \frac{c(t) e^{\frac{2 n i t}{a}}}{e^{\frac{2(2 n+\kappa) i t}{a}}[a(t)+i b(t)] X^{+}(t)}\left[\cot \frac{t-z}{a}-i\right] \mathrm{d} t \\
& =\frac{2 e^{\frac{2(n+\kappa) i z}{a}} X^{+}(z)}{a \pi} \int_{-\frac{a \pi}{2}}^{\frac{a \pi}{2}} \frac{c(t) e^{\frac{2(n+1) i t}{a}}}{[a(t)+i b(t)] X^{+}(t)} \frac{e^{\frac{-2(2 n+\kappa) i t}{a}}}{e^{\frac{2 i z}{a}}-e^{\frac{2 i i}{a}}} \mathrm{~d} t \\
& =\delta_{1}(z)-\delta_{2}(z), \quad z \in \mathcal{C} \backslash \mathcal{X},
\end{aligned}
$$

where

$$
\begin{aligned}
\delta_{1}(z) & =\frac{2 e^{\frac{2(n+\kappa) i z}{a}} X(z)}{a \pi} \int_{-\frac{a \pi}{2}}^{\frac{a \pi}{2}} \frac{c(t) e^{\frac{2(n+1) i t}{a}}}{[a(t)+i b(t)] X^{+}(t)} \frac{e^{\frac{-2(2 n+\kappa) i z}{a}}}{e^{\frac{2 i z}{a}}-e^{\frac{2 i t}{a}}} \mathrm{~d} t \\
& =\frac{e^{-\frac{2 n i z}{a}} X(z)}{a \pi} \int_{-\frac{a \pi}{2}}^{\frac{a \pi}{2}} \frac{c(t)}{e^{\frac{-2 n i t}{a}}[a(t)+i b(t)] X^{+}(t)} \frac{2 e^{-\frac{2 i t}{a}}}{e^{\frac{2 i z}{a}}-e^{\frac{2 i t}{a}}} \mathrm{~d} t \\
& =\frac{e^{-\frac{2 n i z}{a} X(z)}}{a \pi i} \int_{-\frac{a \pi}{2}}^{\frac{a \pi}{2}} \frac{c(t)}{e^{\frac{-2 n i t}{a}}[a(t)+i b(t)] X^{+}(t)}\left[\cot \frac{t-z}{a}-i\right] \mathrm{d} t
\end{aligned}
$$

and

$$
\begin{aligned}
\delta_{2}(z) & =\frac{2 e^{\frac{2(n+\kappa) i z}{a}}}{a \pi} \int_{-\frac{a \pi}{2}}^{\frac{a \pi}{2}} \frac{c(t) e^{\frac{2(n+1) i t}{a}}}{[a(t)+i b(t)] X^{+}(t)} \frac{e^{-\frac{2(2 n+\kappa) i z}{a}}-e^{\frac{-2(2 n+\kappa) i t}{a}}}{e^{\frac{2 i z}{a}}-e^{\frac{2 i t}{a}}} \mathrm{~d} t \\
& =\frac{2}{a \pi} \sum_{j=0}^{-2 n-\kappa-1}\left[\int_{-\frac{a \pi}{2}}^{\frac{a \pi}{2}} \frac{c(t) e^{\frac{2(n+1) i t}{a}}}{[a(t)+i b(t)] X^{+}(t)} e^{\frac{j i t}{a}} \mathrm{~d} t\right] e^{\frac{(1-j) i z}{a}} \\
& =0 \quad(\text { by }(4.28)) .
\end{aligned}
$$


Thus, by Lemma 4.1, the unique solution of $P H_{n}$ problem (4.1) is

$$
\Phi(z)=\frac{e^{-\frac{2 n i z}{a}} X(z)}{a \pi i} \int_{-\frac{a \pi}{2}}^{\frac{a \pi}{2}} \frac{c(t)}{e^{-\frac{2 n i t}{a}}[a(t)+i b(t)] X^{+}(t)} \cot \frac{t-z}{a} \mathrm{~d} t, \quad z \in \mathcal{Z}^{+} .
$$

Let

$$
W(t)=\frac{1}{\left|X^{+}(t)\right|}
$$

Then, from (4.22) and (4.23), we have

$$
[a(t)+i b(t)] X^{+}(t)=\left|X^{+}(t)\right| e^{i \Theta(t)+i \arg [a(t)+i b(t)]}=i e^{-\frac{\kappa i t}{a}}\left|X^{+}(t)\right| .
$$

Thus, (4.28) becomes

$$
\int_{-\frac{a \pi}{2}}^{\frac{a \pi}{2}} W(t) c(t) e^{\frac{2(j+\ell) i t}{a}} \mathrm{~d} t=0, \quad j=n+1, \ldots,-(n+2 \ell+1)
$$

which is equivalent to the real form

$$
\begin{cases}\int_{-\frac{a \pi}{2}}^{\frac{a \pi}{2}} W(t) c(t) \cos \frac{2 j t}{a} \mathrm{~d} t=0, & j=0, \ldots,-(n+\ell+1), \\ \int_{-\frac{a \pi}{2}}^{\frac{a \pi}{2}} W(t) c(t) \sin \frac{2 j t}{a} \mathrm{~d} t=0, & j=1, \ldots,-(n+\ell+1) .\end{cases}
$$

Theorem 4.1 When $n+\ell \geq 0$ the general solution of $P R_{n, n}$ problem (4.15) is (4.18); when $n+\ell \leq-1, P R_{n, n}$ problem (4.15) has the unique solution (4.27) if and only if the conditions in (4.28) are fulfilled. In one word, the solution of $P R_{n, n}$ problem (4.15) possesses the real degree $2(n+\ell+1)$.

Example 4.2 When $n=0$, we reobtain the results in [7]. Hereby, we directly apply the reflection method based on the principle of the so-called symmetric extension, which seems simpler than that used in [7].

\section{2 $\mathrm{PH}$ problem on the circumferences}

We use the regularization method to solve the $\mathrm{PH}$ problem on the circumferences. In fact, both the reflection method and the regularization method are effective for both the $\mathrm{PH}$ problem on the real axis and the $\mathrm{PH}$ problem on the circumference.

Let $L_{j}=\{z,|z-j a \pi|=r\}$ be the circumferences and $S_{j}^{+}=\mathcal{D}_{j}=\{z,|z-j a \pi|<r\}$, where $r \leq a \pi / 2$. The notations $L$ and $S^{+}$are given by (2.1) and (2.2). In particular, we denote $\mathcal{D}_{0}$ by $\mathcal{D}$.

We consider the following Hilbert boundary problem.

PH problem: Find a periodic holomorphic function $\Phi(z)$ in $S^{+}$and continuous on $\overline{S^{+}}$, with the period $a \pi$, such that

$$
\operatorname{Re}\left\{[a(t)+i b(t)] \Phi^{+}(t)\right\}=c(t), \quad t \in L
$$


where the input functions $a(t), b(t), c(t)$ are periodic real-valued functions with period $a \pi$ on $L$ and $a, b, c \in H(L)$,

$$
|a(t)+b(t)|=1, \quad t \in L \text { (normalized condition). }
$$

Lemma 4.2 (Localization principle [7]) If $\Phi$ is a solution of PH problem (4.37), then its restriction on $\mathcal{D}, \Xi=\left.\Phi\right|_{\mathcal{D}}$, is the solution of the following localization problem. Conversely, if $\Xi$ is the solution of the following localization problem, then its periodic extension will be a solution of $\mathrm{PH}$ problem (4.37):

Localization problem: Find an analytic function $\Xi$ in $\mathcal{D}$ and continuous on $\overline{\mathcal{D}}(\mathcal{D} \cup \partial \mathcal{D})$ such that

$$
\operatorname{Re}\left\{[a(t)+i b(t)] \Xi^{+}(t)\right\}=c(t), \quad t \in \partial \mathcal{D} .
$$

The simplest $P H$ problem is Schwarz problem, i.e., $a(t)+i b(t)=1$. We have the following classical result for its localization problem.

Lemma 4.3 [19] Schwarz problem

$$
\left\{\begin{array}{l}
\operatorname{Re}\left\{\Xi^{+}(t)\right\}=c(t), \quad t \in \partial \mathcal{D} \\
\operatorname{Im}\{\Xi(0)\}=0
\end{array}\right.
$$

has the unique solution

$$
\Xi(z)=\frac{1}{2 \pi i} \int_{\partial \mathcal{D}} c(\tau) \frac{\tau+z}{\tau-z} \frac{\mathrm{d} \tau}{\tau}, \quad z \in \mathcal{D},
$$

which is denoted simply by $\Xi(z)=\mathbf{S}[c](z)$.

Let

$$
\kappa=\frac{1}{2 \pi}\{\arg [a(t)-i b(t)]\}_{\partial \mathcal{D}} .
$$

If $R(t)$ is a positive function defined on $\partial \mathcal{D}$ such that

$$
X^{+}(t)=i R(t)[a(t)-i b(t)], \quad t \in \partial \mathcal{D},
$$

is the positive boundary value of certain function $X$, analytic in $\mathcal{D} \backslash\{0\}$ with the zero of order $\kappa$ at the origin and $X(z) \neq 0$ when $z \in D \backslash\{0\}$ and $X^{+}(t) \neq 0$ when $t \in \partial \mathcal{D}$, then we call, respectively, $R$ and $X$ the regularized factor and the canonical function of $P H$ problem (4.37). There exist surely such a regularized factor and a canonical function of $P H$ problem (4.37). For example, taking

$$
X(z)=i z^{\kappa} \exp \{i \mathbf{S}[\Theta](z)\}, \quad z \in \mathcal{D} \backslash\{0\},
$$

where

$$
\Theta(t)=\arg \left\{t^{-\kappa}[a(t)-i b(t)]\right\}, \quad t \in \partial \mathcal{D} .
$$


Then, by the Plemelj formula, we have

$$
\left\{\begin{array}{l}
R(t)=\frac{X^{+}(t)}{i[a(t)-i b(t)]}=r^{\kappa} \exp \left\{\frac{1}{2 \pi} \int_{\partial D} \Theta(\tau) \frac{\tau+t}{\tau-t} \frac{\mathrm{d} \tau}{\tau}\right\}, \quad t \in \partial D, \\
R\left(r e^{i \theta}\right)=r^{\kappa} \exp \left\{-\frac{1}{2 \pi} \int_{-\pi}^{\pi} \Theta\left(r e^{i \zeta}\right) \cot \frac{\zeta-\theta}{2} \mathrm{~d} \zeta\right\}, \quad-\pi \leq \theta \leq \pi .
\end{array}\right.
$$

So, $R$ and $X$ are just a regularized factor and a canonical function of $P H$ problem (4.37).

Remark 4.1 The integral in the second equality of (4.46) is understood as the principal value integral with Hilbert kernel and at $\theta= \pm \pi$ as

$$
\int_{-\pi}^{\pi} f(\zeta) \cot \frac{\zeta}{2} \mathrm{~d} \zeta=\lim _{\delta \rightarrow 0^{+}} \int_{-\pi+\delta}^{\pi-\delta} f(\zeta) \cot \frac{\zeta}{2} \mathrm{~d} \zeta
$$

when $f$ is Hölder-continuous function with period $2 \pi$ (see [15]).

Let

$$
\varphi(z)=\frac{i \Xi(z)}{X(z)}, \quad z \in \mathcal{D} .
$$

Lemma 4.4 Under (4.48), the localization problem (4.39) is equivalent to the following boundary value problem, i.e. the so-called $S_{k}$ problem.

$S_{\kappa}$ problem: Find an analytic function $\varphi(z)$ in $\mathcal{D} \backslash\{0\}$ and continuous on $\overline{\mathcal{D}}$ such that

$$
\left\{\begin{array}{l}
\operatorname{Re}\left\{\varphi^{+}(t)\right\}=\frac{c(t)}{R(t)}, \quad t \in \partial \mathcal{D} \\
\operatorname{Ord}[\varphi](0) \leq \kappa
\end{array}\right.
$$

where $R$ is the regularized factor given in (4.46).

We solve $S_{\kappa}$ problem by two steps.

Step 1. We consider the following homogeneous problem of (4.49):

$$
\left\{\begin{array}{l}
\operatorname{Re}\left\{\varphi^{+}(t)\right\}=0, \quad t \in \partial \mathcal{D} \\
\operatorname{Ord}[\varphi](0) \leq \kappa
\end{array}\right.
$$

Suppose the principal part of $\varphi$ at the origin $z=0$ is

$$
P . P[\varphi](z)=\left\{\begin{array}{ll}
\sum_{j=0}^{k} a_{j} r^{j} z^{-j}, & \text { if } k \geq 0, \\
0, & \text { if } k<0,
\end{array} \quad z \neq 0 .\right.
$$

Introduce the following symmetric operator:

$$
\mathcal{L}[\varphi](z)= \begin{cases}\operatorname{Re}\left(a_{0}\right)+\sum_{j=1}^{k}\left[a_{j} r^{j} z^{-j}+\overline{a_{j}} r^{-j} z^{j}\right], & \text { if } k>0, \\ \operatorname{Re}\left(a_{0}\right), & \text { if } k=0, \quad z \neq 0 . \\ 0, & \text { if } k<0,\end{cases}
$$

Clearly

$$
\operatorname{Re}\{i \mathcal{L}[\varphi](t)\}=0 \quad \text { when } t \in \partial \mathcal{D} \text {. }
$$


Setting

$$
F(z)=i[\varphi(z)-\mathcal{L}[\varphi](z)], \quad z \in D,
$$

by (4.50) and (4.53),

$$
\begin{cases}\operatorname{Re}\left\{F^{+}(t)\right\}=0, & \text { if } t \in \partial D \\ \operatorname{Im}\{F(z)\}=0, & \text { if } z=0\end{cases}
$$

By Lemma 4.3, $F(z) \equiv 0$. Thus, the general solution of the homogeneous problem (4.50) is

$$
\varphi(z)=\mathcal{L}[\varphi](z), \quad z \in D
$$

Introduce the class of the symmetric Laurent polynomials

$$
S \Pi_{k}= \begin{cases}\left\{q_{k}(z)=\sum_{j=-k}^{k} c_{j} r^{-j} z^{j}: c_{j}=\overline{c_{-j}} \text { for } j=0,1,2, \ldots, k\right\}, & \text { if } k \geq 0, \\ \{0\}, & \text { if } k<0 .\end{cases}
$$

Obviously,

$$
\mathcal{L}[\varphi] \in S \Pi_{\kappa} \quad \text { and } \quad\left\{S \Pi_{\kappa}(t), t \in \partial D\right\} \subset \mathcal{R} \text { (real set) }
$$

thus, we have the following conclusion.

Proposition 4.1 The set of solution of the homogeneous problem (4.50) is $S \Pi_{\kappa}$.

Step 2 . When $\kappa \geq 0$, it is obvious that

$$
\varphi(z)=\frac{1}{2 \pi i} \int_{\partial D} \frac{c(\tau)}{R(\tau)} \frac{\tau+z}{\tau-z} \frac{\mathrm{d} \tau}{\tau}=\frac{1}{2 \pi} \int_{\partial D} \frac{c(\tau)}{[a(\tau)+i b(\tau)] X^{+}(\tau)} \frac{\tau+z}{\tau-z} \frac{\mathrm{d} \tau}{\tau}, \quad z \in \mathcal{D},
$$

is a special solution of $S_{\kappa}$ problem (4.49). When $\kappa<0, S_{\kappa}$ problem is obviously a Schwarz problem (4.40). So, its solution must be (4.59) by Lemma 4.3. On the other hand, (4.59) is the solution of $S_{\kappa}$ problem (4.49) if and only if it has the zero $z=0$ of, at least, order $-\kappa$, which is to say that if and only if

$$
\int_{\partial D} \frac{c(\tau)}{[a(\tau)+i b(\tau)] X^{+}(\tau)} \frac{\mathrm{d} \tau}{\tau^{j}}=0, \quad j=1, \ldots,-\kappa,
$$

$S_{\kappa}$ problem (4.49) has the unique solution (4.59).

Proposition 4.2 If $\kappa \geq 0, S_{\kappa}$ problem (4.49) has the special solution (4.59). If $\kappa<0$, it has the unique solution (4.59) if and only if the condition of solvability (4.60) is satisfied.

Now, for the localization problem (4.39), we easily obtain the following result by Lemma 4.4, Proposition 4.1, and Proposition 4.2 (cf. see [7, 20]): 
Lemma 4.5 When $\kappa \geq 0$, the localization problem (4.39) is solvable and its solution is given by

$$
\begin{aligned}
& \Xi(z)=\frac{X(z)}{2 \pi i}\left[\int_{\partial D} \frac{c(\tau)}{[a(\tau)+i b(\tau)] X^{+}(\tau)} \frac{\tau+z}{\tau-z} \frac{\mathrm{d} \tau}{\tau}+2 \pi i q_{\kappa}(z)\right] \\
& \text { with } q_{\kappa} \in S \Pi_{\kappa}, z \in \mathcal{D} .
\end{aligned}
$$

When $\kappa<0$, the localization problem (4.39) is solvable if and only if the condition of solvability (4.60) is satisfied, and in such a case its solution is given by (4.61) with $q_{\kappa}=0$.

Remark 4.2 The canonical function (4.44) may be rewritten as

$$
X(z)=z^{\kappa} Y(z) \quad \text { with } Y(z)=\exp \{\Gamma(z)\}
$$

where

$$
\begin{aligned}
\Gamma(z) & =\frac{1}{2 \pi i} \int_{\partial \mathcal{D}} \log \left\{\tau^{-\kappa}[a(\tau)-i b(\tau)]\right\} \frac{\tau+z}{\tau-z} \frac{\mathrm{d} \tau}{\tau}+\frac{\pi i}{2} \\
& =\frac{1}{\pi} \int_{\partial \mathcal{D}} \frac{\Theta(\tau)}{\tau-z} \mathrm{~d} \tau-\frac{1}{2 \pi} \int_{\partial \mathcal{D}} \frac{\Theta(\tau)}{\tau} \mathrm{d} \tau+\frac{\pi i}{2} \\
& \equiv \frac{1}{\pi} \int_{\partial \mathcal{D}} \frac{\Theta(\tau)}{\tau-z} \mathrm{~d} \tau+C^{\star} \quad\left(C^{*} \text { a constant }\right) .
\end{aligned}
$$

In [7], the localization problem (4.39) is solved by the method of symmetric extension. Obviously, $Y$ is just the restricted function on $\mathcal{D}$ of the corresponding canonical function used in [7].

Remark 4.3 When $\kappa<0$, the expression of the solution (4.61) may be further simplified. It may be written as

$$
\Xi(z)=\frac{Y(z)}{\pi i} \int_{\partial D} \frac{c(\tau)}{[a(\tau)+i b(\tau)] Y^{+}(\tau)} \frac{\mathrm{d} \tau}{\tau-z}, \quad z \in \mathcal{D} .
$$

In fact,

$$
\begin{aligned}
\Xi(z) & =\frac{X(z)}{2 \pi i} \int_{\partial D} \frac{c(\tau)}{[a(\tau)+i b(\tau)] X^{+}(\tau)} \frac{\tau+z}{\tau-z} \frac{\mathrm{d} \tau}{\tau} \\
& =\frac{z^{\kappa} Y(z)}{2 \pi i} \int_{\partial D} \frac{c(\tau)}{[a(\tau)+i b(\tau)] X^{+}(\tau)}\left[1+\frac{2 z}{\tau-z}\right] \frac{\mathrm{d} \tau}{\tau} \\
& =\frac{Y(z)}{\pi i} \int_{\partial D} \frac{c(\tau)}{[a(\tau)+i b(\tau)] Y^{+}(\tau)} \frac{z^{\kappa+1}}{\tau^{\kappa+1}} \frac{\mathrm{d} \tau}{\tau-z} \quad(\text { by }(4.60)) \\
& =\frac{z^{\kappa+1} Y(z)}{\pi i} \int_{\partial D} \frac{c(\tau)}{[a(\tau)+i b(\tau)] Y^{+}(\tau)}\left[\frac{\tau^{-\kappa-1}-z^{-\kappa-1}}{\tau-z}+\frac{z^{-\kappa-1}}{\tau-z}\right] \mathrm{d} \tau \\
& =\frac{Y(z)}{\pi i} \int_{\partial D} \frac{c(\tau)}{[a(\tau)+i b(\tau)] Y^{+}(\tau)} \frac{\mathrm{d} \tau}{\tau-z} \quad(\text { by }(4.60)) .
\end{aligned}
$$

Remark 4.4 Observe that, by (4.61),

$$
W(\theta)=\exp \left\{-\frac{1}{2 \pi} \int_{0}^{2 \pi} \Theta\left(e^{i \zeta}\right) \cot \frac{\zeta-\theta}{2} \mathrm{~d} \zeta\right\}, \quad-\pi \leq \theta \leq \pi,
$$


is the positive, $2 \pi$-periodic continuous function, which is called the weight function, then (4.60) is equivalent to the following real conditions:

$$
\begin{cases}\int_{-\pi}^{\pi} W(\theta) c\left(r e^{i \theta}\right) \sin j \theta \mathrm{d} \theta=0, & j=1,2, \ldots,-\kappa-1, \\ \int_{-\pi}^{\pi} W(\theta) c\left(r e^{i \theta}\right) \cos j \theta \mathrm{d} \theta=0, & j=0,1,2, \ldots,-\kappa-1,\end{cases}
$$

which indicate that $c\left(e^{i \theta}\right)$ is orthogonal to $H_{n}^{T}=\left\{T_{n}: T_{n}(\theta)=\sum_{k=0}^{n}\left(a_{k} \sin k \theta+b_{k} \cos k \theta\right)\right\}$ with the weight function $W$, which just shows that the real degree of freedom of the solution for the localization problem (4.39) is $2 \kappa+1$.

In order to obtain the solution of $P H$ problem (4.37), we only need to set up the periodic extension of (4.61), which is rather simple.

Let

$$
\Phi(z)=\Xi(z-j a \pi), \quad z \in S_{j}^{+}(j=0, \pm 1, \pm 2, \ldots) .
$$

Now, by Lemma 4.2 and Lemma 4.5, Remark 4.3, and Remark 4.4, we finally have the following result.

Theorem 4.2 When $\kappa \geq 0, P H$ problem (4.37) is solvable and its general solution may be written as

$$
\begin{gathered}
\Phi(z)=\frac{X(z-j a \pi)}{2 \pi i}\left[\int_{\partial D} \frac{c(\tau)}{[a(\tau)+i b(\tau)] X^{+}(\tau)} \frac{\tau+(z-j a \pi)}{\tau-(z-j a \pi)} \frac{\mathrm{d} \tau}{\tau}+q_{\kappa}(z-j a \pi)\right], \\
z \in \mathcal{D}_{j}(j=0, \pm 1, \pm 2, \ldots) \text { with } q_{\kappa} \in S \Pi_{\kappa} .
\end{gathered}
$$

When $\kappa<0$, if and only if the condition of solvability

$$
\int_{\partial D} \frac{c(\tau)}{[a(\tau)+i b(\tau)] X^{+}(\tau)} \frac{\mathrm{d} \tau}{\tau^{j}}=0, \quad j=1, \ldots,-\kappa,
$$

is satisfied, or, $-2 \kappa-1$ real conditions in (4.67) are satisfied, $P H$ problem (4.37) is solvable and its solution is given by

$$
\begin{aligned}
\Phi(z) & =\frac{X(z-j a \pi)}{2 \pi i} \int_{\partial D} \frac{c(\tau)}{[a(\tau)+i b(\tau)] X^{+}(\tau)} \frac{\tau+(z-j a \pi)}{\tau-(z-j a \pi)} \frac{\mathrm{d} \tau}{\tau}, \\
z & \in \mathcal{D}_{j}(j=0, \pm 1, \pm 2, \ldots),
\end{aligned}
$$

or

$$
\begin{aligned}
\Phi(z) & =\frac{Y(z-j a \pi)}{\pi i} \int_{\partial D} \frac{c(\tau)}{[a(\tau)+i b(\tau)] Y^{+}(\tau)} \frac{\mathrm{d} \tau}{\tau-(z-j a \pi)}, \\
z \in \mathcal{D}_{j}(j=0, \pm 1, \pm 2, \ldots) &
\end{aligned}
$$

where $Y$ is given by (4.62). 
Authors' contributions

All authors contributed equally and significantly in writing this article. All authors read and approved the final manuscript.

\section{Author details}

${ }^{1}$ Faculty of Information Technology, Macau University of Science and Technology, Macao, P.R. China. ${ }^{2}$ School of Science, Linyi University, Linyi, Shandong 276000, P.R. China. ${ }^{3}$ Department of Mathematics, Wuhan University, Wuhan, 430072,

P.R. China. ${ }^{4}$ Department of Mathematics, University of Macau, P.O. Box 3001, Macau (via Hong Kong), P.R. China.

\section{Acknowledgements}

The authors would like to thank the referee for his valuable suggestions, which helped us to improve the presentation of the paper. This work is supported by Macao Science and Technology Development Fund, MSAR. Ref. 018/2014/A1, FDCT of Macao (MSAR Ref. 098/2012/A3), Multi-Year Research Grant (MYRG) MYRG115(Y1-L4)-FST13-QT, NNSF of China (\#11171260), DYSP and AMEP of Linyi University.

Received: 17 April 2015 Accepted: 4 August 2015 Published online: 19 August 2015

\section{References}

1. Lu, JK: Complex Variable Methods in Plane Elasticity. World Scientific, Singapore (1995)

2. Muskhelishvili, NI: Some Basic Problems of Mathematical Theory of Elasticity. Noordhoff, Groningen (1963)

3. Al-Smadi, M, Abu Arqub, O, Momani, S: A computational method for two-point boundary value problems of fourth-order mixed integrodifferential equations. Math. Probl. Eng. 2013, Article ID 832074 (2013)

4. Abu-Gdairi, R, Al-Smadi, M: An efficient computational method for 4th-order boundary value problems of Fredholm IDEs. Appl. Math. Sci. (Ruse) 7(93-96), 4761-4774 (2013)

5. Al-Smadi, M, Abu Arqub, O, El-Ajou, A: A numerical iterative method for solving systems of first-order periodic boundary value problems. J. Appl. Math. 2014, Article ID 135465 (2014)

6. Abu Arqub, O, Al-Smadi, M: Numerical algorithm for solving two-point, second-order periodic boundary value problems for mixed integro-differential equations. Appl. Math. Comput. 243, 911-922 (2014)

7. Lu, JK: Boundary Value Problems for Analytic Functions. World Scientific, Singapore (1993)

8. Muskhelishvili, NI: Singular Integral Equations, 2nd edn. Noordhoff, Groningen (1968)

9. Gakhov, FD: Boundary Problems. Nauka, Moscow (1977)

10. Chibrikova, Ll: On Riemann boundary problem for automorphic functions. J. Kazan Univ. 116(4), 59-109 (1956)

11. Lu, JK: Periodic Riemann boundary value problems and their applications to elasticity. Chin. Math. 4, 372-422 (1964)

12. Lu, JK: On fundamental problems of plane elasticity with periodic stresses. Acta Mech. Sin. 7(4), 316-327 (1964)

13. Cai, HT, Lu, JK: Mathematical Theory in Periodic Plane Elasticity. Gordon \& Breach, Singapore (2000)

14. Du, JY: Some systems of orthogonal trigonometric polynomials associated with singular integrals with Hilbert kernel. J. Wuhan Univ. Natur. Sci. Ed. 2(2), 17-35 (1988)

15. Du, JY: On the collocation methods for singular integral equations with Hilbert kernel. Math. Comput. 78(266), 891-928 (2009)

16. $\mathrm{Du}, \mathrm{JY}, \mathrm{Han}, \mathrm{HL}$, Jin, QX: On trigonometric and paratrigonometric Hermite interpolation. J. Approx. Theory 131(1), 74-99 (2004)

17. Wang, XY, Du, JY: Periodic Riemann boundary value problems. China Science Paper Online. http://www.paper.edu.cn

18. Du, JY, Wang, YF: On boundary value problem of polyanalytic function on the real axis. Complex Var. Theory Appl. 48(6), 527-542 (2003)

19. Wang, YF, Du, JY: Hilbert boundary value problems of polyanalytic functions on the unit circumference. Complex Var. Elliptic Equ. 51(8-11), 923-943 (2006)

20. Mshimba, AS: On the Hilbert boundary value problem for holomorphic function in the Sobolev spaces. Appl. Anal. 30, 87-99 (1988)

\section{Submit your manuscript to a SpringerOpen ${ }^{\circ}$ journal and benefit from:}

- Convenient online submission

Rigorous peer review

- Immediate publication on acceptance

- Open access: articles freely available online

- High visibility within the field

- Retaining the copyright to your article 